ESAIM: M2AN 48 (2014) 1451-1472

DOI: $10.1051 / \mathrm{m} 2 \mathrm{an} / 2014003$
ESAIM: Mathematical Modelling and Numerical Analysis

www.esaim-m2an.org

\title{
MATHEMATICAL ANALYSIS OF A DISCRETE FRACTURE MODEL COUPLING DARCY FLOW IN THE MATRIX WITH DARCY-FORCHHEIMER FLOW IN THE FRACTURE*
}

\author{
Peter Knabner ${ }^{1}$ and Jean E. Roberts ${ }^{2}$
}

\begin{abstract}
We consider a model for flow in a porous medium with a fracture in which the flow in the fracture is governed by the Darcy-Forchheimer law while that in the surrounding matrix is governed by Darcy's law. We give an appropriate mixed, variational formulation and show existence and uniqueness of the solution. To show existence we give an analogous formulation for the model in which the Darcy-Forchheimer law is the governing equation throughout the domain. We show existence and uniqueness of the solution and show that the solution for the model with Darcy's law in the matrix is the weak limit of solutions of the model with the Darcy-Forchheimer law in the entire domain when the Forchheimer coefficient in the matrix tends toward zero.
\end{abstract}

Mathematics Subject Classification. 35J60, 76S05.

Received March 21, 2012.

Published online August 13, 2014.

\section{INTRODUCTION}

Numerical modeling of fluid flow in a porous medium, even single-phase, incompressible fluid flow, is complicated because the permeability coefficient characterizing the medium may vary over several orders of magnitude within a region quite small in comparison to the dimensions of the domain. This is in particular the case when fractures are present in the medium. Fractures have at least one dimension that is very small, much smaller than a reasonable discretization parameter given the size of the domain, but are much more permeable (or possibly, due to crystalization, much less permeable) than the surrounding medium. They thus have a very significant influence on the fluid flow but adapting a standard finite element or finite volume mesh to handle flow in the fractures poses obvious problems. Many models have been developed to study fluid flow in porous media with fractures. Models may employ a continuum representation of fractures as in the double porosity models derived by homogenization or they may be discrete fracture models. Among the discrete fracture models are models of discrete fracture networks in which only the flow in the fractures is considered. The more complex discrete

Keywords and phrases. Flow in porous media, fractures, Darcy-Forchheimer flow, solvability, regularization, monotone operators.

* Franco-German cooporation program PHC Procope.

1 University of Erlangen-Nuremberg, Department of Mathematics, Cauerstr. 11, 91058 Erlangen, Germany.

knabner@am. uni-erlangen. de

2 Inria Paris-Rocquencourt, B.P. 105, 78153 Le Chesnay, France. jean.roberts@inria.fr 
fracture models couple flow in the fractures or in fracture networks with flow in the surrounding medium. This later type model is the type considered here.

An alternative to the possibility of using a very fine grid in the fracture and a necessarily much coarser grid away from the fracture is the possibility of treating the fracture as an $(n-1)$-dimensional hypersurface in the $n$-dimensional porous medium. This is the idea that was developed in [2] for highly permeable fractures and in [16] for fractures that may be highly permeable or nearly impermeable. Similar models have also been studied in $[6,11,17]$. These articles were all concerned with the case of single-phase, incompressible flow governed by Darcy's law and the law of mass conservation. In [14] a model was derived in which Darcy's law was replaced by the Darcy-Forchheimer law for the flow in the fracture, while Darcy's law was maintained for flow in the rest of the medium. The model was approximated numerically with mixed finite elements and some numerical experiments were carried out.

The use of the linear Darcy law as the constitutive law for fluid flow in porous media, together with the continuity equation, is well established. For medium-ranged velocities it fits well with experiments ([8], Chapter 5) and can be derived rigorously (on simpler periodic media) by homogenization starting from Stokes's equation $[3,4,19]$. However, for high velocities experiments show deviations which indicate the need for a nonlinear correction term $([8,12]$, Chapter 5). The simplest proposed is a term quadratic in velocity, the Forchheimer correction. In fractured media, the permeability (or hydraulic conductivity) in the fractures is generally much greater than in the surrounding medium so that the total flow process in the limit is dominated by the fracture flow. This indicates that a modeling different from Darcy's model is necessary and leads us to investigate models combining Darcy and Darcy-Forchheimer flow.

In this paper we consider existence and uniqueness of the solution of corresponding stationery problems. Assumptions on coefficients should be weak so as not to prevent the use of the results in more complex real life situations. Therefore we aim at weak solutions of an appropriate variational formulation, where we prefer a mixed variational formulation, due to the structure of the problems and a further use of mixed finite element techniques. For a simple d-dimensional domain $\Omega$ and for the linear Darcy flow the results are well known (cf. [9]) and rely on the coercivity of the operator $A$ coming from Darcy's equation on the kernel of the divergence operator $B$ coming from the continuity equation and the functional setting in $H(\operatorname{div}, \Omega)$ for the flux and $L^{2}(\Omega)$ for the pressure. For the nonlinear Darcy-Forchheimer flow the functional setting has to be changed to $W^{3}(\operatorname{div}, \Omega)$ (see Appendix A.1) for the flux so that $A$ will remain (strictly) monotone and to $L^{\frac{3}{2}}(\Omega)$ for the pressure. This makes it possible to extend the reasoning for the linear case to the homogeneous Darcy-Forchheimer problem and via regularization, using the Browder-Minty theorem for maximal monotone operators, also to prove unique existence in the inhomogeneous case. This work is carried out in the thesis [18]; see also $[5,10,15]$ for related results. Here we extend this reasoning to the situation of two subdomains of the matrix separated by a fracture with various choices of the constitutive laws in domains and fractures. One would expect that the Darcy-Forchheimer law is more accurate than Darcy's law (and this will be partially made rigorous); therefore, (and for technical reasons) we start with a model having the Darcy-Forchheimer law throughout the domain (though with strongly variable coefficients) and extend the aforementioned reasoning for existence and uniqueness to this case, (Sect. 3). By its derivation, Darcy's law should be a limit case of the Darcy-Forchheimer law. This is made precise in Section 4 by showing that the solution of the Darcy model is a weak limit of solutions of the Darcy-Forchheimer model with the Forchheimer coefficient (multiplying the nonlinear term) going to 0 . This was shown earlier in [5] under slightly different assumptions, but we include it here for completeness. This opens up the possibility of treating various combinations of the constitutive laws. As rapid transport is more likely to take place in the fractures, we explicitly treat the case of Darcy's law in the matrix and the Darcy-Forchheimer law in fractures. By using the full Darcy-Forchheimer model as a regularization and deriving corresponding a priori bounds we can show the existence of a solution as a weak limit of the regularizing full models (Sect. 5). Uniqueness again follows as in all the other cases from the monotone structure of the problem (see Appendix A.2). Technical difficulties stem from the different functional settings for the linear case and the nonlinear case. It may be envisaged to extend this basic procedure in various directions. An obvious extension is to the case of a finite number of fractures and subdomains, as long as the 
fractures do not intersect, which is quite restrictive. But also a general case where d-dimensional subdomains are separated by (d-1)-dimensional fractures, which are separated by (d-2)-dimensional fractures, etc. may be attacked with this approach. Another extension could be the investigation of other nonlinear correction terms to Darcy's law: $c f$. [7].

The outline of this article is as follows: in Section 2 the model problem with Darcy flow in the matrix and Darcy-Forchheimer flow in the fracture as well as the problem with Darcy-Forchheimer flow in the matrix and in the fracture will be given. In Section 3 the existence and uniqueness of the solution to the problem with Darcy-Forchheimer flow in the matrix and in the fracture will be shown. Section 4 is concerned with showing that in a simple domain (one without a fracture) that the solution of the Darcy problem is obtained as the limit of the Darcy-Forchheimer problem when the Forchheimer coefficient tends to zero. Then Section 5 takes up the problem for extending the result of Section 4 to the case of a domain with a fracture in which it is shown that the problem with Darcy-Forchheimer flow in the fracture but with Darcy flow in the matrix is obtained as the limit of the problem with Darcy-Forchheimer flow everywhere as the Forchheimer coefficient in the matrix tends to zero.

\section{Formulation of the Problems}

\subsection{Formulation with Darcy-Forchheimer flow in the fracture and darcy flow in the matrix}

Let $\Omega$ be a bounded domain in $R^{d}$ with boundary $\Gamma$, and let $\gamma \subset \Omega$ be a $(d-1)$-dimensional surface that separates $\Omega$ into two subdomains: $\Omega \subset R^{d}, \quad \Omega=\Omega_{1} \cup \gamma \cup \Omega_{2}, \quad \gamma=\left(\bar{\Omega}_{1} \cap \bar{\Omega}_{2}\right) \cap \Omega, \quad \Gamma=\partial \Omega$, and $\quad \Gamma_{i}=$ $\Gamma \cap \partial \Omega_{i}$. We suppose for simplicity that $\gamma$ is a subset of a hyperplane; i.e. that $\gamma$ is flat. Taking the stratification of natural porous media into account this seems to be a feasible assumption covering a variety of situations. The extension to the case that $\gamma$ is a smooth surface should not pose any major problems but would be considerably more complex as the curvature tensor would enter into the definitions of the tangential gradient and the tangential divergence. We consider the following problem, which was derived in $[13,14]$ :

$$
\begin{aligned}
\alpha_{i} \mathbf{u}_{i}+\nabla p_{i} & =0 & & \text { in } \Omega_{i} \\
\operatorname{div} \mathbf{u}_{i} & =q_{i} & & \text { in } \Omega_{i} \\
p_{i} & =p_{d, i} & & \text { on } \Gamma_{i}
\end{aligned}
$$

together with

$$
\begin{aligned}
\left(\alpha_{\gamma}+\beta_{\gamma}\left|\mathbf{u}_{\gamma}\right|\right) \mathbf{u}_{\gamma}+\nabla p_{\gamma} & =0 & & \text { on } \gamma \\
\operatorname{div} \mathbf{u}_{\gamma} & =q_{\gamma}+\left[\mathbf{u}_{1} \cdot \mathbf{n}-\mathbf{u}_{2} \cdot \mathbf{n}\right] & & \text { on } \gamma \\
p_{\gamma} & =p_{d, \gamma} & & \text { on } \partial \gamma
\end{aligned}
$$

and the interface condition

$$
p_{i}=p_{\gamma}+(-1)^{i+1} \kappa\left(\xi \mathbf{u}_{i} \cdot \mathbf{n}+\bar{\xi} \mathbf{u}_{i+1} \cdot \mathbf{n}\right), \quad \text { on } \gamma, \quad i=1,2,
$$

where $\mathbf{n}$ is the unit normal vector on $\gamma$, directed outward from $\Omega_{1}, \kappa$ is a coefficient function on $\gamma$ related directly to the fracture width and inversely to the normal component of the permeability of the physical fracture, the parameter $\xi$ is a constant greater than $1 / 2$ and $\bar{\xi}=1-\xi$, and for convenience of notation the index $i$ of the subdomains is considered to be an element of $Z_{2}$ (so that if $i=2$, then $i+1=1$ ). The tensor coefficients $\alpha_{i}, i=1,2$, and $\alpha_{\gamma}$ are related to the inverse of the permeability tensors on $\Omega_{i}, i=1,2$, and $\gamma$, respectively, and the coefficient $\beta_{\gamma}$ is the Forchheimer coefficient on $\gamma$, assumed to be scalar. We assume that the functions $\alpha_{i}: \Omega_{i} \longrightarrow R^{d, d}, \quad \alpha_{\gamma}: \gamma \longrightarrow R^{d-1, d-1}$, are all symmetric and uniformly positive definite:

$$
\begin{array}{rlrlrl}
\underline{\alpha}_{i}|\mathbf{x}|^{2} & \leq \mathbf{x} \cdot \alpha_{i}(\mathbf{y}) \mathbf{x} & \leq \bar{\alpha}_{i}|\mathbf{x}|^{2} & & \forall \mathbf{y} \in \Omega_{i}, \quad \mathbf{x} \in R^{d} \\
\text { and } & \underline{\alpha}_{\gamma}|\mathbf{x}|^{2} & \leq \mathbf{x} \cdot \alpha_{\gamma}(\mathbf{y}) \mathbf{x} & \leq \bar{\alpha}_{\gamma}|\mathbf{x}|^{2} & & \forall \mathbf{y} \in \gamma, \quad \mathbf{x} \in R^{d-1} \\
\underline{\beta}_{\gamma} & \leq \beta_{\gamma}(\mathbf{y}) \leq \bar{\beta}_{\gamma} & & \forall \mathbf{y} \in \gamma, &
\end{array}
$$


where $\underline{\alpha}_{i}, \underline{\alpha}_{\gamma}, \underline{\beta}_{\gamma}>0$, and that the real valued coefficient function $\kappa: \gamma \longrightarrow R$ is bounded above and below by positive constants:

$$
0\langle\underline{\kappa} \leq \kappa(\mathbf{y}) \leq \bar{\kappa} \quad \forall \mathbf{y} \in \gamma .
$$

Note that only minimal assumptions concerning $\alpha_{i}, i=1,2, \alpha_{\gamma}$ and $\beta_{\gamma}$ reflecting the structure of the problem are required and no further regularity, allowing for general heterogeneous media. However this means that the standard functional setting of the linear case has to be modified and thus also the regularity requirements concerning the source and boundary terms.

We make the following assumptions concerning the data functions $q$ and $p_{d}$ corresponding respectively to an external source term and to Dirichlet boundary data:

$$
\begin{aligned}
q & =\left(q_{1}, q_{2}, q_{\gamma}\right) \in L^{3}\left(\Omega_{1}\right) \times L^{3}\left(\Omega_{2}\right) \times L^{3}(\gamma) \\
p_{d}=\left(p_{d, 1}, p_{d, 2}, p_{d, \gamma}\right) & \in\left(W^{\frac{1}{3}, \frac{3}{2}}\left(\Gamma_{1}\right) \cap H^{\frac{1}{2}}\left(\Gamma_{1}\right)\right) \times\left(W^{\frac{1}{3}, \frac{3}{2}}\left(\Gamma_{2}\right) \cap H^{\frac{1}{2}}\left(\Gamma_{2}\right)\right) \times H^{\frac{1}{2}}(\partial \gamma),
\end{aligned}
$$

where we have used the standard notation for the Lebesgue spaces $L^{p}, p \in R, p \geq 1$, and for the Sobolev spaces $W^{k, p}, k, p \in R, p \geq 1$; see [1]. Following standard practice we often write $H^{k}$ for the Sobolev space $W^{k, 2}, k \in R$. We have required more regularity of the data functions than necessary for a weak formulation of problem (2.1)-(2.3) in order to use the same data functions for problem (2.1)-(2.3) and for problem (2.11)-(2.13) given below.

To give a weak mixed formulation of problem (2.1), (2.2), (2.3), we introduce several spaces of functions:

$$
\begin{gathered}
\mathcal{M}=\left\{p=\left(p_{1}, p_{2}, p_{\gamma}\right): p_{i} \in L^{2}\left(\Omega_{i}\right), i=1,2, \text { and } p_{\gamma} \in L^{3 / 2}(\gamma)\right\} \\
\|p\|_{\mathcal{M}}=\sum_{i=1}^{2}\left\|p_{i}\right\|_{0,2, \Omega_{i}}+\left\|p_{\gamma}\right\|_{0, \frac{3}{2}, \gamma} .
\end{gathered}
$$

The space $\mathcal{M}$ being a product of reflexive Banach spaces is clearly a reflexive Banach space with the dual space

$$
\begin{gathered}
\mathcal{M}^{\prime}=\left\{f=\left(f_{1}, f_{2}, f_{\gamma}\right): f_{i} \in L^{2}\left(\Omega_{i}\right), i=1,2, \text { and } f_{\gamma} \in L^{3}(\gamma)\right\} \\
\|f\|_{\mathcal{M}^{\prime}}=\sum_{i=1}^{2}\left\|f_{i}\right\|_{0,2, \Omega_{i}}+\left\|f_{\gamma}\right\|_{0,3, \gamma}
\end{gathered}
$$

We also define

$$
\begin{gathered}
\mathbf{V}=\left\{\mathbf{v}=\left(\mathbf{v}_{1}, \mathbf{v}_{2}, \mathbf{v}_{\gamma}\right): \mathbf{v}_{i} \in\left(L^{2}\left(\Omega_{i}\right)\right)^{d}, i=1,2, \text { and } \mathbf{v}_{\gamma} \in\left(L^{3}(\gamma)\right)^{d-1}\right\} \\
\|\mathbf{v}\|_{\mathbf{v}}=\sum_{i=1}^{2}\left\|\mathbf{v}_{i}\right\|_{0,2, \Omega_{i}}+\left\|\mathbf{v}_{\gamma}\right\|_{0,3, \gamma}
\end{gathered}
$$

and its dual space

$$
\begin{gathered}
\mathbf{V}^{\prime}=\left\{\mathbf{g}=\left(\mathbf{g}_{1}, \mathbf{g}_{2}, \mathbf{g}_{\gamma}\right): \mathbf{g}_{i} \in\left(L^{2}\left(\Omega_{i}\right)\right)^{d}, i=1,2, \text { and } \mathbf{g}_{\gamma} \in\left(L^{\frac{3}{2}}(\gamma)\right)^{d-1}\right\} \\
\|\mathbf{g}\|_{\mathbf{V}^{\prime}}=\sum_{i=1}^{2}\left\|\mathbf{g}_{i}\right\|_{0,2, \Omega_{i}}+\left\|\mathbf{g}_{\gamma}\right\|_{0, \frac{3}{2}, \gamma}
\end{gathered}
$$

Remark 2.1. For $f=\left(f_{1}, f_{2}, f_{\gamma}\right) \in \mathcal{M}^{\prime}$, respectively $\mathbf{g}=\left(\mathbf{g}_{1}, \mathbf{g}_{2}, \mathbf{g}_{\gamma}\right) \in \mathbf{V}^{\prime}$, we have used the $\ell^{1}$ norm on $R^{3}$ to give the norm of $f$, respectively $\mathbf{g}$, in terms of its three components $f_{1}, f_{2}$ and $f_{\gamma}$, respectively $\mathbf{g}_{1}, \mathbf{g}_{2}$ and $\mathbf{g}_{\gamma}$, whereas the actual norm for the dual space would have used the $\ell^{\infty}$ or maximum norm. However these norms are equivalent since $R^{3}$ is of finite dimension and we have found it more convenient to use the $\ell^{1}$ norms here.

For the domains $\Omega_{1}, \Omega_{2}$ in $R^{d}$ and $\gamma$ in $R^{d-1}$, respectively, we need minimal regularity to make some of the expressions used below well defined. In particular, we need exterior normal vector fields on the boundaries. To 
assume that the domains are Lipschitzian will be sufficient, and this will be done henceforth. We will need in addition the space $\mathbf{W}$ defined by

$$
\begin{aligned}
& \mathbf{W}=\left\{\mathbf{u}=\left(\mathbf{u}_{1}, \mathbf{u}_{2}, \mathbf{u}_{\gamma}\right) \in \mathbf{V}\right.: \operatorname{Div} \mathbf{u}:=\left(\operatorname{div} \mathbf{u}_{1}, \operatorname{div} \mathbf{u}_{2}, \operatorname{div} \mathbf{u}_{\gamma}-\left[\mathbf{u}_{1} \cdot \mathbf{n}-\mathbf{u}_{2} \cdot \mathbf{n}\right]\right) \in \mathcal{M}^{\prime} \\
&\text { and } \left.\mathbf{u}_{i} \cdot \mathbf{n} \in L^{2}(\gamma), i=1,2\right\} \\
&\left.\|\mathbf{u}\|_{\mathbf{W}}=\|\mathbf{u}\|_{\mathbf{V}}+\|\operatorname{Div} \mathbf{u}\|_{\mathcal{M}^{\prime}}+\sum_{i=1}^{2}\left\|\mathbf{u}_{i} \cdot \mathbf{n}\right\|_{0,2, \gamma}\right)
\end{aligned}
$$

One can show that $\mathbf{W}$ is also a reflexive Banach space and that

$$
\mathcal{D}=\left(\mathcal{D}\left(\bar{\Omega}_{1}\right)\right)^{d} \times\left(\mathcal{D}\left(\bar{\Omega}_{2}\right)\right)^{d} \times(\mathcal{D}(\bar{\gamma}))^{d-1}
$$

is dense in $\mathbf{W}$ (see e.g. [18], Lem. 3.13), where by $\mathcal{D}(\overline{\mathcal{O}})$ is meant $\left\{\psi_{\mid \mathcal{O}}: \psi \in C^{\infty}\left(R^{n}\right)\right\}$, for $\mathcal{O}$ a bounded domain in $R^{n}$. We also have that for $\mathbf{v} \in \mathbf{W}, \mathbf{v}_{i} \in H\left(\operatorname{div}, \Omega_{i}\right), i=1,2$, and $\mathbf{v}_{\gamma} \in H(\operatorname{div}, \gamma)\left(\right.$ since $\left.L^{3}(\gamma) \subset L^{2}(\gamma)\right)$ so that $\mathbf{v}_{i} \cdot \mathbf{n}_{i} \in H^{-\frac{1}{2}}\left(\partial \Omega_{i}\right)$ and $\mathbf{v}_{\gamma} \cdot \mathbf{n}_{\gamma} \in H^{-\frac{1}{2}}(\partial \gamma)$, where $\mathbf{n}_{i}, i=1,2$, and $\mathbf{n}_{\gamma}$ are the exterior normal vectors on $\partial \Omega_{i}, i=1,2$, and on $\partial \gamma$, respectively.

Define the forms $a: \mathbf{W} \times \mathbf{W} \longrightarrow R$ and $b: \mathbf{W} \times \mathcal{M} \longrightarrow R$ by

$$
\begin{aligned}
& a(\mathbf{u}, \mathbf{v})=\sum_{i=1}^{2} \int_{\Omega_{i}} \alpha_{i} \mathbf{u}_{i} \cdot \mathbf{v}_{i} \mathrm{~d} x+\int_{\gamma}\left(\alpha_{\gamma}+\beta_{\gamma}\left|\mathbf{u}_{\gamma}\right|\right) \mathbf{u}_{\gamma} \cdot \mathbf{v}_{\gamma} \mathrm{d} s+\sum_{i=1}^{2} \int_{\gamma} \kappa\left(\xi \mathbf{u}_{i} \cdot \mathbf{n}+\bar{\xi} \mathbf{u}_{i+1} \cdot \mathbf{n}\right) \mathbf{v}_{i} \cdot \mathbf{n} \mathrm{d} s \\
& b(\mathbf{u}, r)=\sum_{i=1}^{2} \int_{\Omega_{i}} \operatorname{div} \mathbf{u}_{i} r_{i} \mathrm{~d} x+\int_{\gamma}\left(\operatorname{div} \mathbf{u}_{\gamma}-\left[\mathbf{u}_{1} \cdot \mathbf{n}-\mathbf{u}_{2} \cdot \mathbf{n}\right]\right) r_{\gamma} \mathrm{d} s=\langle\operatorname{Div} \mathbf{u}, r\rangle_{\mathcal{M}^{\prime}, \mathcal{M}} .
\end{aligned}
$$

Note that the form $a$ is continuous and linear in its second variable while the form $b$ is clearly continuous and bilinear. Define the continuous, linear forms $\mathbf{g} \in \mathbf{W}^{\prime}$ and $f \in \mathcal{M}^{\prime}$ by

$$
\begin{aligned}
& \mathrm{g}: \mathrm{W} \longrightarrow R \\
& \mathbf{g}(\mathbf{v})=-\sum_{i=1}^{2}\left\langle p_{d, i}, \mathbf{v}_{i} \cdot \mathbf{n}_{i}\right\rangle_{H^{\frac{1}{2}}\left(\Gamma_{i}\right), H^{-\frac{1}{2}}\left(\Gamma_{i}\right)}-\left\langle p_{d, \gamma}, \mathbf{v}_{\gamma} \cdot \mathbf{n}_{\gamma}\right\rangle_{H^{\frac{1}{2}}(\partial \gamma), H^{-\frac{1}{2}}(\partial \gamma)} \\
& \text { and } f: \mathcal{M} \longrightarrow R \\
& f(r)=\sum_{i=1}^{2} \int_{\Omega_{i}} q_{i} r_{i} \mathrm{~d} x+\int_{\gamma} q_{\gamma} r_{\gamma} \mathrm{d} s .
\end{aligned}
$$

The weak mixed formulation of (2.1), (2.2) and (2.3) is

Find $\mathbf{u} \in \mathbf{W}$ and $p \in \mathcal{M}$ such that

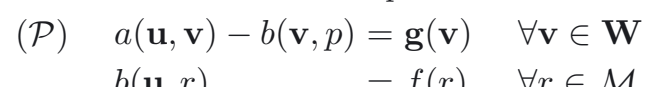

Define also, for the moment only formally (see Lem. 3.1),

$$
\begin{aligned}
& A: \mathbf{W} \longrightarrow \mathbf{W}^{\prime} \\
& \langle A(\mathbf{u}), \mathbf{v}\rangle_{\mathbf{W}^{\prime}, \mathbf{W}}=a(\mathbf{u}, \mathbf{v}) \quad \forall \mathbf{v} \in \mathbf{W}
\end{aligned} \quad \text { and } \quad \begin{aligned}
& B: \mathbf{W} \longrightarrow \mathcal{M}^{\prime} \\
& \\
& \langle B(\mathbf{u}), r\rangle_{\mathcal{M}^{\prime}, \mathcal{M}}=b(\mathbf{u}, r) \quad \forall r \in \mathcal{M}
\end{aligned}
$$

and note that $B: \mathbf{W} \longrightarrow \mathcal{M}^{\prime}$ is simply Div $: \mathbf{W} \longrightarrow \mathcal{M}^{\prime}$ so that for $\widetilde{\mathbf{W}}$, the kernel of $B$,

$$
\widetilde{\mathbf{W}}=\left\{\mathbf{v}=\left(\mathbf{v}_{1}, \mathbf{v}_{2}, \mathbf{v}_{\gamma}\right) \in \mathbf{W}: \operatorname{Div} \mathbf{v}=B(\mathbf{v})=0\right\},
$$

we have that

$$
\|\mathbf{v}\|_{\mathbf{w}}=\|\mathbf{v}\|_{\mathbf{v}}+\sum_{i=1}^{2}\left\|\mathbf{v}_{i} \cdot \mathbf{n}\right\|_{0,2, \gamma} \quad \forall \mathbf{v} \in \widetilde{\mathbf{W}}
$$




\subsection{Formulation with Darcy-Forchheimer flow in the matrix and in the fractures}

With $\Omega, \gamma, \Omega_{i}, \Gamma_{i}, \mathbf{n}_{i}, i=1,2, \mathbf{n}_{\gamma}$ and $\mathbf{n}$ as well as $\alpha_{i}, \alpha_{\gamma}, \beta_{\gamma}, \kappa, q_{i}, q_{\gamma}, p_{d, i}, p_{d, \gamma}, \xi$, and $\bar{\xi}$ as in the preceding paragraph, and with $\beta_{i}: \Omega_{i} \longrightarrow R$ a function satisfying

$$
\underline{\beta}_{i} \leq \beta_{i}(\mathbf{y}) \leq \bar{\beta}_{i} \quad \forall \mathbf{y} \in \Omega_{i},
$$

where $\left.\underline{\beta}_{i}, \bar{\beta}_{i}\right\rangle 0$, we now consider the following problem:

$$
\begin{aligned}
\left(\alpha_{i}+\beta_{i}\left|\mathbf{u}_{i}\right|\right) \mathbf{u}_{i}+\nabla p_{i} & =0 & & \text { in } \Omega_{i} \\
\operatorname{div} \mathbf{u}_{i} & =q_{i} & & \text { in } \Omega_{i} \\
p_{i} & =p_{d, i} & & \text { on } \Gamma_{i}
\end{aligned}
$$

together with

$$
\begin{aligned}
\left(\alpha_{\gamma}+\beta_{\gamma}\left|\mathbf{u}_{\gamma}\right|\right) \mathbf{u}_{\gamma}+\nabla p_{\gamma} & =0 & & \text { on } \gamma \\
\operatorname{div} \mathbf{u}_{\gamma} & =q_{\gamma}+\left[\mathbf{u}_{1} \cdot \mathbf{n}-\mathbf{u}_{2} \cdot \mathbf{n}\right] & & \text { on } \gamma \\
p_{\gamma} & =p_{d, \gamma} & & \text { on } \partial \gamma
\end{aligned}
$$

and the interface conditions

$$
p_{i}=p_{\gamma}+(-1)^{i+1} \kappa\left(\xi \mathbf{u}_{i} \cdot \mathbf{n}+\bar{\xi} \mathbf{u}_{i+1} \cdot \mathbf{n}\right), \quad i=1,2 .
$$

Due to the Forchheimer regularization in the matrix equations, the spaces in the earlier definitions need to be replaced by spaces appropriate for the functional setting of the Forchheimer equations, i.e. $L^{2}\left(\Omega_{i}\right)$ by $L^{\frac{3}{2}}\left(\Omega_{i}\right)$, and consequently $L^{2}\left(\Omega_{i}\right)$ by $L^{3}\left(\Omega_{i}\right)$ for the dual spaces, thus obtaining a $\beta$-version of the earlier spaces, i.e. $\mathcal{M}_{\beta}$ instead of $\mathcal{M}$, etc. For the sake of clarity we state explicitly:

$$
\begin{gathered}
\mathcal{M}_{\beta}=\left\{p=\left(p_{1}, p_{2}, p_{\gamma}\right): p_{i} \in L^{3 / 2}\left(\Omega_{i}\right), i=1,2, \text { and } p_{\gamma} \in L^{3 / 2}(\gamma)\right\} \\
\|p\|_{\mathcal{M}_{\beta}}=\sum_{i=1}^{2}\left\|p_{i}\right\|_{0, \frac{3}{2}, \Omega_{i}}+\left\|p_{\gamma}\right\|_{0, \frac{3}{2}, \gamma}
\end{gathered}
$$

The space $\mathcal{M}_{\beta}$ is clearly a reflexive Banach space with dual space

$$
\begin{gathered}
\mathcal{M}_{\beta}^{\prime}=\left\{f=\left(f_{1}, f_{2}, f_{\gamma}\right): f_{i} \in L^{3}\left(\Omega_{i}\right), i=1,2, \text { and } f_{\gamma} \in L^{3}(\gamma)\right\} \\
\|f\|_{\mathcal{M}_{\beta}^{\prime}}=\sum_{i=1}^{2}\left\|f_{i}\right\|_{0,3, \Omega_{i}}+\left\|f_{\gamma}\right\|_{0,3, \gamma} .
\end{gathered}
$$

We also define

$$
\begin{gathered}
\mathbf{V}_{\beta}=\left\{\mathbf{v}=\left(\mathbf{v}_{1}, \mathbf{v}_{2}, \mathbf{v}_{\gamma}\right): \mathbf{v}_{i} \in\left(L^{3}\left(\Omega_{i}\right)\right)^{d}, i=1,2, \text { and } \mathbf{v}_{\gamma} \in\left(L^{3}(\gamma)\right)^{d-1}\right\} \\
\|\mathbf{v}\|_{\mathbf{v}_{\beta}}=\sum_{i=1}^{2}\left\|\mathbf{v}_{i}\right\|_{0,3, \Omega_{i}}+\left\|\mathbf{v}_{\gamma}\right\|_{0,3, \gamma},
\end{gathered}
$$

which is similarly a reflexive Banach space, with its dual space

$$
\begin{gathered}
\mathbf{V}_{\beta}^{\prime}=\left\{\mathbf{g}=\left(\mathbf{g}_{1}, \mathbf{g}_{2}, \mathbf{g}_{\gamma}\right): \mathbf{g}_{i} \in\left(L^{3 / 2}\left(\Omega_{i}\right)\right)^{d}, i=1,2, \text { and } \mathbf{g}_{\gamma} \in\left(L^{3 / 2}(\gamma)\right)^{d-1}\right\} \\
\|\mathbf{g}\|_{\mathbf{V}_{\beta}^{\prime}}=\sum_{i=1}^{2}\left\|\mathbf{g}_{i}\right\|_{0, \frac{3}{2}, \Omega_{i}}+\left\|\mathbf{g}_{\gamma}\right\|_{0, \frac{3}{2}, \gamma} .
\end{gathered}
$$

Again we have used the equivalent $\ell^{1}$ norm instead of the $\ell^{\infty}$ norm to construct the product space norm for $\mathcal{M}_{\beta}^{\prime}$ and $\mathbf{W}_{\beta}^{\prime}$. We also need the space $\mathbf{W}_{\beta}$ defined by

$$
\begin{gathered}
\mathbf{W}_{\beta}=\left\{\mathbf{u}=\left(\mathbf{u}_{1}, \mathbf{u}_{2}, \mathbf{u}_{\gamma}\right) \in \mathbf{V}_{\beta}: \operatorname{Div} \mathbf{u}=\left(\operatorname{div} \mathbf{u}_{1}, \operatorname{div} \mathbf{u}_{2}, \operatorname{div} \mathbf{u}_{\gamma}-\left[\mathbf{u}_{1} \cdot \mathbf{n}-\mathbf{u}_{2} \cdot \mathbf{n}\right]\right) \in \mathcal{M}_{\beta}^{\prime}\right. \\
\left.\quad \text { and } \mathbf{u}_{i} \cdot \mathbf{n} \in L^{2}(\gamma), i=1,2\right\} \\
\|\mathbf{u}\|_{\mathbf{W}_{\beta}}=\|\mathbf{u}\|_{\mathbf{V}_{\beta}}+\|\operatorname{Div} \mathbf{u}\|_{\mathcal{M}_{\beta}^{\prime}}+\sum_{i=1}^{2}\left\|\mathbf{u}_{i} \cdot \mathbf{n}\right\|_{0,2, \gamma} .
\end{gathered}
$$


One can show that $\mathbf{W}_{\beta}$ is a reflexive Banach space, that $\mathcal{D}$, given by (2.8), is dense in $\mathbf{W}_{\beta}$, that for $\mathbf{v} \in \mathbf{W}_{\beta}$, for $i=1,2, \mathbf{v}_{i} \in H\left(\operatorname{div}, \Omega_{i}\right)$ and $\mathbf{v}_{\gamma} \in H(\operatorname{div}, \gamma)$ so that $\mathbf{v}_{i} \cdot \mathbf{n}_{i} \in H^{-\frac{1}{2}}\left(\partial \Omega_{i}\right)$ and $\mathbf{v}_{\gamma} \cdot \mathbf{n}_{\gamma} \in H^{-\frac{1}{2}}(\partial \gamma)$. Further $\mathbf{v}_{i} \in W^{3}\left(\operatorname{div}, \Omega_{i}\right)$ (see Appendix A.1). Define the forms $a_{\beta}: \mathbf{W}_{\beta} \times \mathbf{W}_{\beta} \longrightarrow R$ and $b_{\beta}: \mathbf{W}_{\beta} \times \mathcal{M}_{\beta} \longrightarrow R$ by

$$
\begin{aligned}
& a_{\beta}(\mathbf{u}, \mathbf{v})=\sum_{i=1}^{2} \int_{\Omega_{i}}\left(\alpha_{i}+\beta_{i}\left|\mathbf{u}_{i}\right|\right) \mathbf{u}_{i} \cdot \mathbf{v}_{i} \mathrm{~d} x+\int_{\gamma}\left(\alpha_{\gamma}+\beta_{\gamma}\left|\mathbf{u}_{\gamma}\right|\right) \mathbf{u}_{\gamma} \cdot \mathbf{v}_{\gamma} \mathrm{d} s+\sum_{i=1}^{2} \int_{\gamma} \kappa\left(\xi \mathbf{u}_{i} \cdot \mathbf{n}+\bar{\xi} \mathbf{u}_{i+1} \cdot \mathbf{n}\right) \mathbf{v}_{i} \cdot \mathbf{n} \mathrm{d} s \\
& b_{\beta}(\mathbf{u}, r)=\sum_{i=1}^{2} \int_{\Omega_{i}} \operatorname{div} \mathbf{u}_{i} r_{i} \mathrm{~d} x+\int_{\gamma}\left(\operatorname{div} \mathbf{u}_{\gamma}-\left[\mathbf{u}_{1} \cdot \mathbf{n}-\mathbf{u}_{2} \cdot \mathbf{n}\right]\right) r_{\gamma} \mathrm{d} s=\langle\operatorname{Div} \mathbf{u}, r\rangle_{\mathcal{M}_{\beta}^{\prime}, \mathcal{M}_{\beta}} .
\end{aligned}
$$

Note that the form $a_{\beta}$ is continunous and linear in its second variable while $b_{\beta}$ is continuous and bilinear. Define the linear forms

$$
\mathrm{g}: \mathbf{W}_{\beta} \longrightarrow R \text { and } f: \mathcal{M}_{\beta} \longrightarrow R
$$

as in (2.9) but with $\mathbf{g} \in \mathbf{W}_{\beta}^{\prime}$ and $f \in \mathcal{M}_{\beta}^{\prime}$ which is valid with the regularity assumptions in (2.6). The mixed weak formulation of (2.11), (2.12) and (2.13) is given by

$$
\begin{aligned}
& \text { Find } \mathbf{u} \in \mathbf{W}_{\beta} \text { and } p \in \mathcal{M}_{\beta} \text { such that } \\
& \left(\mathcal{P}_{\beta}\right) \quad a_{\beta}(\mathbf{u}, \mathbf{v})-b_{\beta}(\mathbf{v}, p)=\mathbf{g}(\mathbf{v}) \quad \forall \mathbf{v} \in \mathbf{W}_{\beta} \\
& b_{\beta}(\mathbf{u}, r) \quad=f(r) \quad \forall r \in \mathcal{M}_{\beta} \text {. }
\end{aligned}
$$

Define again

$$
\begin{array}{lll}
A_{\beta}: \mathbf{W}_{\beta} \longrightarrow \mathbf{W}_{\beta}^{\prime} & \text { and } \quad \begin{array}{l}
B_{\beta}: \mathbf{W}_{\beta} \longrightarrow \mathcal{M}_{\beta}^{\prime} \\
\left\langle A_{\beta}(\mathbf{u}), \mathbf{v}\right\rangle_{\mathbf{V}^{\prime}}, \mathbf{v}=a_{\beta}(\mathbf{u}, \mathbf{v}) \quad \forall \mathbf{v} \in \mathbf{W}_{\beta}
\end{array} \quad \begin{array}{l}
\left\langle B_{\beta}(\mathbf{u}), r\right\rangle_{\mathcal{M}_{\beta}^{\prime}}, \mathcal{M}_{\beta} \\
\end{array} \quad b_{\beta}(\mathbf{u}, r) \quad \forall r \in \mathcal{M}_{\beta}
\end{array}
$$

for an equivalent operator equation and

$$
\widetilde{\mathbf{W}}_{\beta}=\left\{\mathbf{u}=\left(\mathbf{u}_{1}, \mathbf{u}_{2}, \mathbf{u}_{\gamma}\right) \in \mathbf{W}_{\beta}: \operatorname{Div} \mathbf{u}:=B_{\beta}(\mathbf{u})=0\right\}
$$

and note that

$$
\|\mathbf{u}\|_{\mathbf{W}_{\beta}}=\|\mathbf{u}\|_{\mathbf{V}_{\beta}}+\sum_{i=1}^{2}\left\|\mathbf{u}_{i} \cdot \mathbf{n}\right\|_{0,2, \gamma} \quad \forall \mathbf{u} \in \widetilde{\mathbf{W}}_{\beta}
$$

Remark 2.2. Note that none of the spaces $\mathbf{W}_{\beta}, \mathbf{V}_{\beta}, \widetilde{\mathbf{W}}_{\beta}$, or $\mathcal{M}_{\beta}$ and neither of the operators $b_{\beta}$ nor $B_{\beta}$ depends on the coefficient $\beta$. The index $\beta$ is used simply to indicate that these are the spaces and operators used to define the problem $\left(\mathcal{P}_{\beta}\right)$.

To obtain some of the estimates that we will derive in the following sections we shall make use of the following technical lemma given in ([15], Lem. 1.1 and 1.4).

Lemma 2.3. For $\mathbf{x}$ and $\mathbf{y}$ in $R^{n}$, we have the following inequalities:

$$
\begin{aligned}
|| \mathbf{x}|\mathbf{x}-| \mathbf{y}|\mathbf{y}| & \leq(|\mathbf{x}|+|\mathbf{y}|)|\mathbf{x}-\mathbf{y}| \\
\frac{1}{2}|\mathbf{x}-\mathbf{y}|^{3} & \leq(|\mathbf{x}| \mathbf{x}-|\mathbf{y}| \mathbf{y}) \cdot(\mathbf{x}-\mathbf{y}), \\
\left.|| \mathbf{x}\right|^{-\frac{1}{2}} \mathbf{x}-|\mathbf{y}|^{-\frac{1}{2}} \mathbf{y} \mid & \leq \sqrt{2}|\mathbf{x}-\mathbf{y}|^{\frac{1}{2}} \\
\frac{|\mathbf{x}-\mathbf{y}|^{2}}{\sqrt{|\mathbf{x}|}+\sqrt{|\mathbf{y}|}} & \leq\left(\frac{\mathbf{x}}{\sqrt{|\mathbf{x}|}}-\frac{\mathbf{y}}{\sqrt{|\mathbf{y}|}}\right) \cdot(\mathbf{x}-\mathbf{y}) .
\end{aligned}
$$


In (2.18) and hereafter $x \mapsto|x|^{-\frac{1}{2}} x$ on $R^{n}$ means the continuation of this function on $R^{n} \backslash\{0\}$ to $R^{n}$ obtained by defining $|0|^{-\frac{1}{2}} 0:=0$, which by $(2.18)$ is indeed Hölder continuous with exponent $\frac{1}{2}$.

Here we introduce some notation that we will use throughout the remainder of the article: for any positive integer $n$ and any bounded domain $\mathcal{O}$ in $R^{n}$, we know that $L^{3}(\mathcal{O}) \hookrightarrow L^{2}(\mathcal{O})$ and that the inclusion map is continuous so that there is a constant $C_{L, \mathcal{O}}$ depending on $n$ and the measure of the space such that if $\phi \in L^{3}(\mathcal{O})$ then $\|\phi\|_{L^{2}} \leq C_{L, \mathcal{O}}\|\phi\|_{L^{3}}$. Here we shall assume that $C_{L}$ is a constant with $C_{L, \mathcal{O}} \leq C_{L}$ for all of the spaces $\mathcal{O}$ that we deal with. (There are only a finite number for each problem.) Also we know that if $s$ and $t$ are such that $1 \leq s \leq t \leq \infty$ then the $\ell^{s}$ and $\ell^{t}$ norms on $R^{n}$ are equivalent (since all norms on finite dimensional spaces are equivalent), and we shall assume that there are positive real numbers $C_{\ell}$ and $c_{\ell}$ such that if $\mathrm{x} \in R^{n}$ then $c_{\ell}\|\mathbf{x}\|_{\ell^{t}} \leq\|\mathbf{x}\|_{\ell^{s}} \leq C_{\ell}\|\mathbf{x}\|_{\ell^{t}}$ for all dimensions $n$ and all norms $\ell^{s}$ and $\ell^{t}$ with $1 \leq s \leq t \leq \infty$, that we encounter in the problems that follow. (Again there will only be a finite number.)

\section{Existence And Uniqueness of the SOlution of The PRoblem $\left(\mathcal{P}_{\beta}\right)$ DARCY-FORCHHEIMER FLOW IN THE FRACTURE AND IN THE SUBDOMAINS}

To show the existence and uniqueness of the solution of $\left(\mathcal{P}_{\beta}\right)$, following the argument of [15], Section 1 we show that the operator $A_{\beta}: \mathbf{W}_{\beta} \longrightarrow \mathbf{W}_{\beta}^{\prime}$ is continuous and monotone and is uniformly monotone on $\widetilde{\mathbf{W}}_{\beta}$ to obtain a solution to the homogeneous problem with $f=0$. (That $B_{\beta}: \mathbf{W}_{\beta} \longrightarrow \mathcal{M}_{\beta}^{\prime}$ satisfies the inf-sup condition follows just as in the linear case, $c f$. [16], however for completeness a demonstration is given in Appendix A.3). Then taking any solution to the second equation of $\left(\mathcal{P}_{\beta}\right)$ (whose existence is guaranteed by the inf-sup condition) an auxiliary homogeneous problem is constructed whose solution can be used to produce the solution of $\left(\mathcal{P}_{\beta}\right)$.

Lemma 3.1. The operator $A_{\beta}: \mathbf{W}_{\beta} \longrightarrow \mathbf{W}_{\beta}^{\prime}$ is continuous and strictly monotone and is furthermore uniformly monotone on $\widetilde{\mathbf{W}}_{\beta}$.

Proof. To see that $\forall \mathbf{u} \in \mathbf{W}_{\beta}, A_{\beta}(\mathbf{u}) \in \mathbf{W}_{\beta}^{\prime}$ i.e. that $\forall \mathbf{u} \in \mathbf{W}_{\beta}, A_{\beta}(\mathbf{u})$ is bounded, suppose that $\mathbf{u} \in \mathbf{W}_{\beta}$. Then, using the equivalence of norms in a finite dimensional space and Hölder's inequality, we have, for each $\mathbf{v}$ in $\mathbf{W}_{\beta}$,

$$
\begin{aligned}
\int_{\Omega_{i}} \beta_{i}\left|\mathbf{u}_{i}\right| \mathbf{u}_{i} \cdot \mathbf{v}_{i} \mathrm{~d} x & \leq \bar{\beta}_{i} C_{\ell}^{3} \int_{\Omega_{i}}\left|\mathbf{u}_{i}\right|_{3}^{2}\left|\mathbf{v}_{i}\right|_{3} \mathrm{~d} x \leq \bar{\beta}_{i} C_{\ell}^{3}\left(\int_{\Omega_{i}}\left(\left|\mathbf{u}_{i}\right|_{3}^{2}\right)^{\frac{3}{2}} \mathrm{~d} s\right)^{\frac{2}{3}}\left(\int_{\Omega_{i}}\left|\mathbf{v}_{i}\right|_{3}^{3} \mathrm{~d} s\right)^{\frac{1}{3}} \\
& \leq \bar{\beta}_{i} C_{\ell}^{3}\left\|\mathbf{u}_{i}\right\|_{0,3, \Omega_{i}}^{2}\left\|\mathbf{v}_{i}\right\|_{0,3, \Omega_{i}} .
\end{aligned}
$$

We also have

$$
\int_{\Omega_{i}} \alpha_{i} \mathbf{u}_{i} \cdot \mathbf{v}_{i} \mathrm{~d} x \leq \bar{\alpha}_{i}\left\|\mathbf{u}_{i}\right\|_{0,2, \Omega_{i}}\left\|\mathbf{v}_{i}\right\|_{0,2, \Omega_{i}} \leq \bar{\alpha}_{i} C_{L}^{2}\left\|\mathbf{u}_{i}\right\|_{0,3, \Omega_{i}}\left\|\mathbf{v}_{i}\right\|_{0,3, \Omega_{i}}
$$

As we have similar inequalities for the norms on $\gamma$ and as $\xi \geq \bar{\xi}$ we conclude that for each $\mathbf{v} \in \mathbf{W}_{\beta}$,

$$
\begin{aligned}
\left\|A_{\beta}(\mathbf{u})\right\| \mathbf{w}_{\beta}^{\prime}= & \sup _{\substack{\mathbf{v} \in \mathbf{w}_{\beta} \\
\mathbf{v} \neq 0}} \frac{\left|\left\langle A_{\beta}(\mathbf{u}), \mathbf{v}\right\rangle_{\mathbf{w}_{\beta}^{\prime}, \mathbf{w}_{\beta}}\right|}{\|\mathbf{v}\|_{\mathbf{w}_{\beta}}} \\
\leq & \sup _{\substack{\mathbf{v} \in \mathbf{w}_{\beta} \\
\mathbf{v} \neq 0}}\left\{\sum_{i=1}^{2}\left(\bar{\alpha}_{i} C_{L}^{2}\left\|\mathbf{u}_{i}\right\|_{0,3, \Omega_{i}}\left\|\mathbf{v}_{i}\right\|_{0,3, \Omega_{i}}+\bar{\beta}_{i} C_{\ell}^{3}\left\|\mathbf{u}_{i}\right\|_{0,3, \Omega_{i}}^{2}\left\|\mathbf{v}_{i}\right\|_{0,3, \Omega_{i}}\right)\right. \\
& +\bar{\alpha}_{\gamma} C_{L}^{2}\left\|\mathbf{u}_{\gamma}\right\|_{0,3, \gamma}\left\|\mathbf{v}_{\gamma}\right\|_{0,3, \gamma}+\bar{\beta}_{\gamma} C_{\ell}^{3}\left\|\mathbf{u}_{\gamma}\right\|_{0,3, \gamma}^{2}\left\|\mathbf{v}_{\gamma}\right\|_{0,3, \gamma} \\
& \left.+\xi \bar{\kappa}\left(\sum_{i=1}^{2}\left\|\mathbf{u}_{i} \cdot \mathbf{n}\right\|_{0,2, \gamma}\right)\left(\sum_{i=1}^{2}\left\|\mathbf{v}_{i} \cdot \mathbf{n}\right\|_{0,2, \gamma}\right)\right\} /\|\mathbf{v}\|_{\mathbf{w}_{\beta}}
\end{aligned}
$$




$$
\begin{aligned}
\leq & \sum_{i=1}^{2}\left(\bar{\alpha}_{i} C_{L}^{2}\left\|\mathbf{u}_{i}\right\|_{0,3, \Omega_{i}}+\bar{\beta}_{i} C_{\ell}^{3}\left\|\mathbf{u}_{i}\right\|_{0,3, \Omega_{i}}^{2}\right) \\
& +\bar{\alpha}_{\gamma} C_{L}^{2}\left\|\mathbf{u}_{\gamma}\right\|_{0,3, \gamma}+\bar{\beta}_{\gamma} C_{\ell}^{3}\left\|\mathbf{u}_{\gamma}\right\|_{0,3, \gamma}^{2}+\xi \bar{\kappa} \sum_{i=1}^{2}\left\|\mathbf{u}_{i} \cdot \mathbf{n}\right\|_{0,2, \gamma} \\
\leq & \bar{\alpha} C_{L}^{2}\|\mathbf{u}\|_{\mathbf{v}_{\beta}}+\bar{\beta} C_{\ell}^{3}\|\mathbf{u}\|_{\mathbf{V}_{\beta}}^{2}+\xi \bar{\kappa} \sum_{i=1}^{2}\left\|\mathbf{u}_{i} \cdot \mathbf{n}\right\|_{0,2, \gamma} \\
\leq & \left(\max \left\{\bar{\alpha} C_{L}^{2}, \xi \bar{\kappa}\right\}+\bar{\beta} C_{\ell}^{3}\|\mathbf{u}\|_{\mathbf{v}_{\beta}}\right)\|\mathbf{u}\|_{\mathbf{w}_{\beta}},
\end{aligned}
$$

where $\bar{\alpha}$ is the $\max \left\{\bar{\alpha}_{1}, \bar{\alpha}_{2}, \bar{\alpha}_{\gamma}\right\}$, and similarly for $\bar{\beta}$.

To see that $A_{\beta}: \mathbf{W}_{\beta} \longrightarrow \mathbf{W}_{\beta}^{\prime}$ is continuous suppose that $\mathbf{u}$ and $\mathbf{w}$ are elements of $\mathbf{W}_{\beta}$. Using Hölder's inequality and then inequality (2.16) along with the equivalence of norms in finite dimensional spaces, we see that, for any $\mathbf{v} \in \mathbf{W}_{\beta}$ and for $i=1,2$,

$$
\begin{aligned}
& \int_{\Omega_{i}} \beta_{i}\left(\left|\mathbf{u}_{i}\right| \mathbf{u}_{i}-\left|\mathbf{w}_{i}\right| \mathbf{w}_{i}\right) \cdot \mathbf{v}_{i} \mathrm{~d} x \leq \bar{\beta}_{i}\left\|\left|\mathbf{u}_{i}\right| \mathbf{u}_{i}-\left|\mathbf{w}_{i}\right| \mathbf{w}_{i}\right\|_{0, \frac{3}{2}, \Omega_{i}}\left\|\mathbf{v}_{i}\right\|_{0,3, \Omega_{i}} \\
& \leq \bar{\beta}_{i} C_{\ell}^{3}\left\|\left(\left\|\mathbf{u}_{i}\right\|_{0,3, \Omega_{i}}+\left\|\mathbf{w}_{i}\right\|_{0,3, \Omega_{i}}\right)\right\| \mathbf{u}_{i}-\mathbf{w}_{i}\left\|_{0,3, \Omega_{i}}\right\| \mathbf{v}_{i} \|_{0,3, \Omega_{i}} .
\end{aligned}
$$

Then using the analogous inequality for the nonlinear term on $\gamma$ we have

$$
\begin{aligned}
\left\|A_{\beta}(\mathbf{u})-A_{\beta}(\mathbf{w})\right\| \mathbf{w}_{\beta}^{\prime}= & \sup _{\substack{\mathbf{v} \in \mathbf{W}_{\beta} \\
\mathbf{v} \neq 0}} \frac{\left\langle A_{\beta}(\mathbf{u})-A_{\beta}(\mathbf{w}), \mathbf{v}\right\rangle_{\mathbf{w}_{\beta}^{\prime}, \mathbf{w}_{\beta}}}{\|\mathbf{v}\|_{\mathbf{w}_{\beta}}} \\
\leq & \left(\left(\bar{\alpha} C_{L}^{2}\|\mathbf{u}-\mathbf{w}\|_{\mathbf{v}_{\beta}}+\bar{\beta} C_{\ell}^{3}\left(\|\mathbf{u}\|_{\mathbf{v}_{\beta}}+\|\mathbf{w}\| \mathbf{v}_{\beta}\right)\|\mathbf{u}-\mathbf{w}\|_{\mathbf{v}_{\beta}}\right)\|\mathbf{v}\|_{\mathbf{v}_{\beta}}\right. \\
& \left.+\xi \bar{\kappa} \sum_{i=1}^{2}\left\|\left(\mathbf{u}_{i}-\mathbf{w}_{i}\right) \cdot \mathbf{n}\right\|_{0,2, \gamma} \sum_{i=1}^{2}\left\|\mathbf{v}_{i} \cdot \mathbf{n}\right\|_{0,2, \gamma}\right) /\|\mathbf{v}\|_{\mathbf{w}_{\beta}} \\
\leq & \left(\max \left\{\bar{\alpha} C_{L}^{2}, \xi \bar{\kappa}\right\}+\bar{\beta} C_{\ell}^{3}\left(\|\mathbf{u}\|_{\mathbf{v}_{\beta}}+\|\mathbf{w}\|_{\mathbf{v}_{\beta}}\right)\right)\|\mathbf{u}-\mathbf{w}\|_{\mathbf{w}_{\beta}} .
\end{aligned}
$$

To see that $A_{\beta}: \mathbf{W}_{\beta} \longrightarrow \mathbf{W}_{\beta}^{\prime}$ is strictly monotone suppose again that $\mathbf{u}$ and $\mathbf{w}$ are elements of $\mathbf{W}_{\beta}$. Then using inequality (2.17), for $i=1,2$,

$$
\int_{\Omega_{i}} \beta_{i}\left(\left|\mathbf{u}_{i}\right| \mathbf{u}_{i}-\left|\mathbf{w}_{i}\right| \mathbf{w}_{i}\right) \cdot\left(\mathbf{u}_{i}-\mathbf{w}_{i}\right) \mathrm{d} x \geq \frac{\beta_{i} c_{\ell}^{3}}{2}\left\|\mathbf{u}_{i}-\mathbf{w}_{i}\right\|_{0,3, \Omega_{i}}^{3} .
$$

We also note that if $x, y \in R$ then $\xi\left(x^{2}+y^{2}\right)+2 \bar{\xi} x y \geq \min \{1,2 \xi-1\}\left(x^{2}+y^{2}\right)$. It follows that $\forall \mathbf{u}, \mathbf{w} \in \mathbf{W}_{\beta}$

$$
\begin{aligned}
\left\langle A_{\beta}(\mathbf{u})-A_{\beta}(\mathbf{w}), \mathbf{u}-\mathbf{w}\right\rangle_{\mathbf{w}_{\beta}^{\prime}, \mathbf{w}_{\beta}} & \geq \frac{\underline{\beta} c_{\ell}^{3}}{2}\|\mathbf{u}-\mathbf{w}\|_{\mathbf{v}_{\beta}}^{3}+\underline{\kappa} \min \{1,2 \xi-1\} \sum_{i=1}^{2}\left\|\left(\mathbf{u}_{i}-\mathbf{w}_{i}\right) \cdot \mathbf{n}\right\|_{0,2, \gamma}^{2} \\
& \geq \mathcal{C}(\underline{\beta}, \underline{\kappa}, \xi)\left(\|\mathbf{u}-\mathbf{w}\|_{\mathbf{v}_{\beta}}^{3}+\sum_{i=1}^{2}\left\|\left(\mathbf{u}_{i}-\mathbf{w}_{i}\right) \cdot \mathbf{n}\right\|_{0,2, \gamma}^{2}\right) \\
& \geq 0,
\end{aligned}
$$

where $\underline{\beta}=\min \left\{\underline{\beta}_{1}, \underline{\beta}_{2}, \underline{\beta}_{\gamma}\right\}$, and where we have equality only if $\mathbf{u}=\mathbf{w}$.

To see that $A_{\beta}$ is uniformly monotone on $\widetilde{\mathbf{W}}_{\beta}$ it suffices to note that if $\mathbf{u}$ and $\mathbf{w}$ belong to $\widetilde{\mathbf{W}}_{\beta}$ then

$$
\left\langle A_{\beta}(\mathbf{u})-A_{\beta}(\mathbf{w}), \mathbf{u}-\mathbf{w}\right\rangle_{\mathbf{w}_{\beta}^{\prime}, \mathbf{w}_{\beta}} \geq \mathcal{G}\left(\|\mathbf{u}-\mathbf{w}\|_{\mathbf{w}_{\beta}}\right)\|\mathbf{u}-\mathbf{w}\|_{\mathbf{w}_{\beta}}
$$


with

$$
\begin{aligned}
\mathcal{G}\left(\|\mathbf{u}\|_{\mathbf{w}_{\beta}}\right):=\mathcal{C}(\underline{\beta}, \underline{\kappa}, \xi) \frac{\|\mathbf{u}\|_{\mathbf{V}_{\beta}}^{3}+\sum_{i=1}^{2}\left\|\mathbf{u}_{i} \cdot \mathbf{n}\right\|_{0,2, \gamma}^{2}}{\|\mathbf{u}\| \mathbf{w}_{\beta}} \\
=\mathcal{C}(\underline{\beta}, \underline{\kappa}, \xi) \frac{\|\mathbf{u}\|_{\mathbf{V}_{\beta}}^{3}+\sum_{i=1}^{2}\left\|\mathbf{u}_{i} \cdot \mathbf{n}\right\|_{0,2, \gamma}^{2}}{\|\mathbf{u}\|_{\mathbf{V}_{\beta}}+\sum_{i=1}^{2}\left\|\mathbf{u}_{i} \cdot \mathbf{n}\right\|_{0,2, \gamma}} \longrightarrow \infty \quad \text { as }\|\mathbf{u}\|_{\mathbf{w}_{\beta}} \longrightarrow \infty,
\end{aligned}
$$

where we have used (2.15).

Lemma 3.2. The linear form $b_{\beta}: \mathbf{W}_{\beta} \times \mathcal{M}_{\beta} \longrightarrow R$ satisfies the following inf-sup condition: there is a positive constant $\theta_{\beta}$ such that $\forall r \in \mathcal{M}_{\beta}$

$$
\theta_{\beta}\|r\|_{\mathcal{M}_{\beta}} \leq \sup _{\mathbf{v} \in \mathbf{W}_{\beta}} \frac{b_{\beta}(\mathbf{v}, r)}{\|\mathbf{v}\|_{\mathbf{W}_{\beta}}}
$$

Proof. See Appendix A.3.

Proposition 3.3. The homogeneous problem

$$
\begin{aligned}
& \text { Find } \mathbf{u}_{\beta}^{0} \in \mathbf{W}_{\beta} \text { and } p_{\beta}^{0} \in \mathcal{M}_{\beta} \text { such that } \\
& \left(\mathcal{P}_{\beta}^{0}\right) \quad a_{\beta}\left(\mathbf{u}_{\beta}^{0}, \mathbf{v}\right)-b_{\beta}\left(\mathbf{v}, p_{\beta}^{0}\right)=\mathbf{g}(\mathbf{v}) \quad \forall \mathbf{v} \in \mathbf{W}_{\beta} \\
& b_{\beta}\left(\mathbf{u}_{\beta}^{0}, r\right) \quad=0 \quad \forall r \in \mathcal{M}_{\beta}
\end{aligned}
$$

has a unique solution.

Proof. That there is a unique solution in $\widetilde{\mathbf{W}}_{\beta}$ to $a_{\beta}\left(\mathbf{u}_{\beta}^{0}, \mathbf{v}\right)=\mathbf{g}(\mathbf{v}), \forall \mathbf{v} \in \widetilde{\mathbf{W}}_{\beta}$, i.e. to $A_{\beta}\left(\mathbf{u}_{\beta}^{0}\right)=\mathbf{g}$, now follows from the Browder-Minty theorem ([20], Thm. 26.A). That there is a unique $p_{\beta}^{0} \in \mathcal{M}_{\beta}$ such that $\left(\mathbf{u}_{\beta}^{0}, p_{\beta}^{0}\right)$ is the unique solution of $\left(\mathcal{P}_{\beta}^{0}\right)$ then follows as in the linear case as the operator $B_{\beta}$ is still linear.

To handle a source term in the continuity equation we start from any solution to this equation and construct an auxiliary homogeneous problem whose solution is then combined with the solution to the (nonhomogeneous) continuity equation to produce the desired solution to the full problem.

Theorem 3.4. The problem $\left(\mathcal{P}_{\beta}\right)$ admits a unique solution $\left(\mathbf{u}_{\beta}, p_{\beta}\right) \in \mathbf{W}_{\beta} \times \mathcal{M}_{\beta}$.

Proof. Since, according to Lemma $3.2, b_{\beta}$ satisfies the inf-sup condition, the subproblem of $\left(\mathcal{P}_{\beta}\right)$

Find $\mathbf{u} \in \mathbf{W}_{\beta}$ such that

$$
b_{\beta}(\mathbf{u}, r)=f(r) \quad \forall r \in \mathcal{M}_{\beta}
$$

has a (non-unique) solution. Let $\mathbf{u}^{*} \in \mathbf{W}_{\beta}$ denote one such. We consider the auxiliary problem

Find $\tilde{\mathbf{u}} \in \mathbf{W}_{\beta}$ and $p \in \mathcal{M}_{\beta}$ such that

$$
\begin{aligned}
& \left(\mathcal{P}_{\beta}^{*}\right) \quad a_{\beta}\left(\tilde{\mathbf{u}}+\mathbf{u}^{*}, \mathbf{v}\right)-b_{\beta}(\mathbf{v}, p)=\mathbf{g}(\mathbf{v}) \quad \forall \mathbf{v} \in \mathbf{W}_{\beta} \\
& b_{\beta}(\tilde{\mathbf{u}}, r) \quad=0 \quad \forall r \in \mathcal{M}_{\beta} .
\end{aligned}
$$

Just as in Proposition 3.3, this problem has a unique solution, as one can show, just as in Lemma 3.1, that

$$
a_{\beta}^{*}(\mathbf{u}, \mathbf{v}):=a_{\beta}\left(\mathbf{u}+\mathbf{u}^{*}, \mathbf{v}\right)
$$

defines a continuous operator, strictly monotone on $\mathbf{W}_{\beta}$ and uniformly monotone on $\widetilde{\mathbf{W}}_{\beta}$. Then, due to the bilinearity of $b_{\beta}, \mathbf{u}:=\tilde{\mathbf{u}}+\mathbf{u}^{*}$, together with $p$ is a solution of $\left(\mathcal{P}_{\beta}\right)$.

To show uniqueness we refer to Lemma A.1 in Appendix A.2. 


\section{Darcy as A limit of DARCy-ForchHeimer - Simple domain}

Suppose here that $\mathcal{O}$ is a bounded domain in $R^{d}$ with boundary $\partial \mathcal{O}$. The object of this section is to show that the solution of the Darcy problem

$$
\begin{array}{cl}
\alpha_{\mathcal{O}} \mathbf{u}=-\nabla p & \text { in } \mathcal{O} \\
\operatorname{div} \mathbf{u}=q_{\mathcal{O}} & \text { in } \mathcal{O} \\
p=p_{\partial, \mathcal{O}} & \text { on } \partial \mathcal{O}
\end{array}
$$

may be obtained as the limit of a sequence of solutions of the Darcy-Forchheimer problems

$$
\begin{array}{cl}
\alpha_{\mathcal{O}} \mathbf{u}_{\beta}+\beta_{\mathcal{O}}\left|\mathbf{u}_{\beta}\right| \mathbf{u}_{\beta}=-\nabla p_{\beta} & \text { in } \mathcal{O} \\
\operatorname{div} \mathbf{u}_{\beta}=q_{\mathcal{O}} & \text { in } \mathcal{O} \\
p_{\beta}=p_{\partial, \mathcal{O}} & \text { on } \partial \mathcal{O}
\end{array}
$$

as $\beta_{\mathcal{O}} \rightarrow 0$. As before we assume that the tensor coefficient function $\alpha_{\mathcal{O}}: \mathcal{O} \longrightarrow R^{d, d}$, is such that

$$
\underline{\alpha}_{\mathcal{O}}|\mathbf{x}|^{2} \leq \mathbf{x} \cdot \alpha_{\mathcal{O}}(\mathbf{y}) \mathbf{x} \leq \bar{\alpha}_{\mathcal{O}}|\mathbf{x}|^{2} \quad \forall \mathbf{y} \in \mathcal{O}, \mathbf{x} \in R^{d},
$$

and the coefficient $\beta_{\mathcal{O}}$ of the nonlinear term is assumed to be a positive real parameter as we are merely interested in obtaining the Darcy problem as a limit of Forchheimer problems. Let

$$
\begin{array}{ll}
\mathbf{W}(\mathcal{O})=H(\operatorname{div}, \mathcal{O}) & \mathcal{M}(\mathcal{O})=L^{2}(\mathcal{O}) \\
\mathbf{W}_{\beta}(\mathcal{O})=W^{3}(\operatorname{div}, \mathcal{O}) & \mathcal{M}_{\beta}(\mathcal{O})=L^{\frac{3}{2}}(\mathcal{O}),
\end{array}
$$

and recall that the image of the normal trace map on $\mathbf{W}(\mathcal{O})$ is $H^{-\frac{1}{2}}(\partial \mathcal{O})$ while the image of the normal trace map on $\mathbf{W}_{\beta}(\mathcal{O})$ is $W^{-\frac{1}{3}, 3}(\partial \mathcal{O})$. Also as before the data functions are assumed to be such that $q \in L^{3}(\mathcal{O})$ and $p_{\partial, \mathcal{O}} \in W^{\frac{1}{3}, \frac{3}{2}}(\partial \mathcal{O}) \cap W^{\frac{1}{2}, 2}(\partial \mathcal{O})$.

Define the bilinear forms $a_{\mathcal{O}}$ and $b_{\mathcal{O}}$ by

$$
\begin{aligned}
& a_{\mathcal{O}}: \mathbf{W}(\mathcal{O}) \times \mathbf{W}(\mathcal{O}) \longrightarrow \quad \text { and } \quad b_{\mathcal{O}}: \mathbf{W}(\mathcal{O}) \times \mathcal{M}(\mathcal{O}) \longrightarrow \quad R \\
& (\mathbf{u}, \mathbf{v}) \mapsto \int_{\mathcal{O}} \alpha_{\mathcal{O}} \mathbf{u} \cdot \mathbf{v} \mathrm{d} x \quad(\mathbf{v}, r) \mapsto \int_{\mathcal{O}} \operatorname{div}(\mathbf{v}) r \mathrm{~d} x,
\end{aligned}
$$

and the linear forms $\mathbf{g}_{\mathcal{O}} \in \mathbf{W}(\mathcal{O})^{\prime}$ and $f_{\mathcal{O}} \in \mathcal{M}(\mathcal{O})^{\prime}$ by

$$
\begin{aligned}
& \mathbf{g}_{\mathcal{O}}: \mathbf{W}(\mathcal{O}) \longrightarrow \quad \text { and } \quad f_{\mathcal{O}}: \mathcal{M}(\mathcal{O}) \longrightarrow \quad R \\
& \mathbf{v} \mapsto\left\langle p_{\partial, \mathcal{O}}, \mathbf{v} \cdot \mathbf{n}\right\rangle_{H^{\frac{1}{2}}(\partial \mathcal{O}), H^{-\frac{1}{2}}(\partial \mathcal{O})} \quad r \mapsto \int_{\mathcal{O}} q_{\mathcal{O}} r \mathrm{~d} x,
\end{aligned}
$$

so that the problem $\left(\mathcal{P}_{\text {Darcy }}\right)$ can be written as

$$
\begin{array}{lll} 
& \text { Find } \mathbf{u} \in \mathbf{W}(\mathcal{O}) \text { and } p \in \mathcal{M}(\mathcal{O}) & \text { such that } \\
\left(\mathcal{P}_{\text {Darcy }}\right) & a_{\mathcal{O}}(\mathbf{u}, \mathbf{v})-b_{\mathcal{O}}(\mathbf{v}, p)=\mathbf{g}_{\mathcal{O}}(\mathbf{v}) & \forall \mathbf{v} \in \mathbf{W}(\mathcal{O}) \\
& b_{\mathcal{O}}(\mathbf{u}, r) \quad=f_{\mathcal{O}}(r) & \forall r \in \mathcal{M}(\mathcal{O}) .
\end{array}
$$

Since $a_{\mathcal{O}}$ is elliptic (coercive) on the subset $\widetilde{\mathbf{W}}(\mathcal{O})=\left\{\mathbf{v} \in \mathbf{W}(\mathcal{O}): b_{\mathcal{O}}(\mathbf{v}, r)=0, \forall r \in \mathcal{M}(\mathcal{O})\right\}$ and $b_{\mathcal{O}}$ satisfies the inf-sup condition on $\mathbf{W}(\mathcal{O}) \times \mathcal{M}(\mathcal{O})$ :

$$
\underline{\alpha}_{\mathcal{O}}\|\mathbf{v}\|_{\mathbf{W}(\mathcal{O})} \leq a_{\mathcal{O}}(\mathbf{v}, \mathbf{v}) \forall \mathbf{v} \in \widetilde{\mathbf{W}}(\mathcal{O}) \quad \text { and } \quad \theta_{\mathcal{O}}\|r\|_{\mathcal{M}(\mathcal{O})} \leq \sup _{\mathbf{v} \in \mathbf{W}(\mathcal{O})} \frac{b_{\mathcal{O}}(\mathbf{v}, r)}{\|\mathbf{v}\|_{\mathbf{W}(\mathcal{O})}}, \forall r \in \mathcal{M}(\mathcal{O}),
$$

the Darcy problem $\left(\mathcal{P}_{\text {Darcy }}\right)$ has a unique solution $\left(\mathbf{u}_{\mathcal{O}}, p_{\mathcal{O}}\right) \in \mathbf{W}(\mathcal{O}) \times \mathcal{M}(\mathcal{O}),[9]$. 
To give the weak formulation of the Forchheimer problem note that since $\mathbf{W}_{\beta}(\mathcal{O}) \subset \mathbf{W}(\mathcal{O})$ the bilinear form $a_{\mathcal{O}}$ is also defined on $\mathbf{W}_{\beta}(\mathcal{O}) \times \mathbf{W}_{\beta}(\mathcal{O})$ and that the bilinear form $b_{\mathcal{O}}$ is also defined on $\mathbf{W}_{\beta}(\mathcal{O}) \times \mathcal{M}_{\beta}(\mathcal{O})$ (even though $\left.\mathcal{M}_{\beta}(\mathcal{O}) \not \subset \mathcal{M}(\mathcal{O})\right)$. Further $b_{\mathcal{O}}$ also satisfies the analogous inf-sup condition on $\mathbf{W}_{\beta}(\mathcal{O}) \times \mathcal{M}_{\beta}(\mathcal{O})$ for some constant $\theta_{\beta, \mathcal{O}}$; see [18] or the more general version in Lemma 3.2. Now define the mapping $a_{\beta, \mathcal{O}}$, linear in its second variable, by

$$
\begin{aligned}
a_{\beta, \mathcal{O}}: \mathbf{W}_{\beta}(\mathcal{O}) \times \mathbf{W}_{\beta}(\mathcal{O}) & \longrightarrow \quad \\
(\mathbf{u}, \mathbf{v}) & \mapsto \int_{\mathcal{O}}\left(\alpha_{\mathcal{O}}+\beta_{\mathcal{O}}|\mathbf{u}|\right) \mathbf{u} \cdot \mathbf{v} \mathrm{d} x,
\end{aligned}
$$

and note that due to the regularity requirements on the data functions $p_{\partial, \mathcal{O}}$ and $q_{\mathcal{O}}$ that the linear forms $\mathbf{g}_{\mathcal{O}}$ and $f_{\mathcal{O}}$ are defined and continuous on $\mathbf{W}_{\beta}(\mathcal{O})$ and $\mathcal{M}_{\beta}(\mathcal{O})$, respectively, (as well as on $\mathbf{W}(\mathcal{O})$ and $\mathcal{M}(\mathcal{O})$ ), so that the problem $\left(\mathcal{P}_{\text {Forch }}\right)$ can be written as

$$
\begin{aligned}
& \text { Find } \mathbf{u}_{\beta} \in \mathbf{W}_{\beta}(\mathcal{O}) \text { and } p_{\beta} \in \mathcal{M}_{\beta}(\mathcal{O}) \text { such that } \\
& \left(\mathcal{P}_{\text {Forch }}\right) \quad a_{\beta, \mathcal{O}}\left(\mathbf{u}_{\beta}, \mathbf{v}\right)-b_{\mathcal{O}}\left(\mathbf{v}, p_{\beta}\right)=\mathbf{g}_{\mathcal{O}}(\mathbf{v}) \quad \forall \mathbf{v} \in \mathbf{W}_{\beta}(\mathcal{O}) \\
& b_{\mathcal{O}}\left(\mathbf{u}_{\beta}, r\right) \quad=f_{\mathcal{O}}(r) \quad \forall r \in \mathcal{M}_{\beta}(\mathcal{O}) \text {. }
\end{aligned}
$$

It is shown in [15] that the form $a_{\beta, \mathcal{O}}$ is continuous, strictly monotone on $\mathbf{W}_{\beta}(\mathcal{O})$, and coercive on $\widetilde{\mathbf{W}}_{\beta}(\mathcal{O})=$ $\left\{\mathbf{v} \in \mathbf{W}_{\beta}(\mathcal{O}): b_{\mathcal{O}}(\mathbf{v}, r)=0, \forall r \in \mathcal{M}_{\beta}(\mathcal{O})\right\}[15]$, Proposition 1.2 and that the Forchheimer problem $\left(\mathcal{P}_{\text {Forch }}\right)$ has a unique solution $\left(\mathbf{u}_{\beta, \mathcal{O}}, p_{\beta, \mathcal{O}}\right) \in \mathbf{W}_{\beta}(\mathcal{O}) \times \mathcal{M}_{\beta}(\mathcal{O})$, [15], Theorem 1.8. Again see the more general vesion of this reasoning in Lemma 3.1.

The demonstration that the solutions of the problems $\left(\mathcal{P}_{\text {Forch }}\right)$ converge to the solution of $\left(\mathcal{P}_{\text {Darcy }}\right)$ is based on a priori bounds for $\mathbf{u}_{\beta, \mathcal{O}}$ and $p_{\beta, \mathcal{O}}$ independent of the parameter $\beta$. In this section we will drop the spaces in the notation for the norms as only $\mathcal{O}$ or $\partial \mathcal{O}$ appears.

Lemma 4.1. There is a constant $C$ independent of $\beta$ such that for $\beta$ sufficiently small

$$
\left\|p_{\beta, \mathcal{O}}\right\|_{\mathcal{M}_{\beta}(\mathcal{O})}+\left\|\mathbf{u}_{\beta, \mathcal{O}}\right\|_{\mathbf{W}(\mathcal{O})}+\beta^{\frac{1}{3}}\left\|\mathbf{u}_{\beta, \mathcal{O}}\right\|_{0,3} \leq C .
$$

In addition,

$$
\beta\left\|\mathbf{u}_{\beta, \mathcal{O}}\right\|_{0,3} \longrightarrow 0, \quad \text { as } \quad \beta \longrightarrow 0 .
$$

Proof. Taking $\mathbf{u}_{\beta, \mathcal{O}}$ for the test function $\mathbf{v}$ in the first equation of $\left(\mathcal{P}_{\text {Forch }}\right)$ and noting that $\mathbf{W}_{\beta}(\mathcal{O}) \subset \mathbf{W}(\mathcal{O})$, as in Section 3 ( $c f$. estimate (3.5)) one obtains

$$
\begin{aligned}
\underline{\alpha}_{\mathcal{O}}\left\|\mathbf{u}_{\beta, \mathcal{O}}\right\|_{0,2}^{2}+\frac{c_{\ell}^{3}}{2} \beta\left\|\mathbf{u}_{\beta, \mathcal{O}}\right\|_{0,3}^{3} & \leq \mathbf{g}_{\mathcal{O}}\left(\mathbf{u}_{\beta, \mathcal{O}}\right)+b\left(\mathbf{u}_{\beta, \mathcal{O}}, p_{\beta, \mathcal{O}}\right) \\
& \leq\left\|\mathbf{g}_{\mathcal{O}}\right\|_{\mathbf{W}(\mathcal{O})^{\prime}}\left\|\mathbf{u}_{\beta, \mathcal{O}}\right\|_{\mathbf{W}(\mathcal{O})}+\left\|\operatorname{div}\left(\mathbf{u}_{\beta, \mathcal{O}}\right)\right\|_{0,3}\left\|p_{\beta, \mathcal{O}}\right\|_{0, \frac{3}{2}} \\
& \leq\left\|\mathbf{g}_{\mathcal{O}}\right\|_{\mathbf{W}(\mathcal{O})^{\prime}}\left(\left\|\mathbf{u}_{\beta, \mathcal{O}}\right\|_{0,2}+\left\|\operatorname{div}\left(\mathbf{u}_{\beta, \mathcal{O}}\right)\right\|_{0,2}\right)+\left\|\operatorname{div}\left(\mathbf{u}_{\beta, \mathcal{O}}\right)\right\|_{0,3}\left\|p_{\beta, \mathcal{O}}\right\|_{0, \frac{3}{2}} .
\end{aligned}
$$

Next directly from the second equation of $\left(\mathcal{P}_{\text {Forch }}\right.$ ) (regarded as an equation in $\mathcal{M}_{\beta}(\mathcal{O})^{\prime}=L^{3}(\mathcal{O})$ ), we obtain

$$
\left\|\operatorname{div}\left(\mathbf{u}_{\beta, \mathcal{O}}\right)\right\|_{0,3}=\left\|f_{\mathcal{O}}\right\|_{0,3},
$$

and, as there is a continuous embedding $\mathcal{M}(\mathcal{O}) \hookrightarrow \mathcal{M}_{\beta}(\mathcal{O})$, i.e. $L^{2}(\mathcal{O}) \hookrightarrow L^{\frac{3}{2}}(\mathcal{O})$ so that the second equation of $\left(\mathcal{P}_{\text {Forch }}\right)$ holds for test functions in $L^{2}(\mathcal{O})$ as well as for those in $L^{\frac{3}{2}}(\mathcal{O})$, we also have

$$
\left\|\operatorname{div}\left(\mathbf{u}_{\beta, \mathcal{O}}\right)\right\|_{0,2}=\left\|f_{\mathcal{O}}\right\|_{0,2} .
$$

Combining these last three inequalities we obtain

$$
\underline{\alpha}_{\mathcal{O}}\left\|\mathbf{u}_{\beta, \mathcal{O}}\right\|_{0,2}^{2}+\frac{c_{\ell}^{3}}{2} \beta\left\|\mathbf{u}_{\beta, \mathcal{O}}\right\|_{0,3}^{3} \leq\left\|\mathbf{g}_{\mathcal{O}}\right\| \mathbf{W}(\mathcal{O})^{\prime}\left(\left\|\mathbf{u}_{\beta, \mathcal{O}}\right\|_{0,2}+\left\|f_{\mathcal{O}}\right\|_{0,2}\right)+\left\|f_{\mathcal{O}}\right\|_{0,3}\left\|p_{\beta, \mathcal{O}}\right\|_{0, \frac{3}{2}}
$$


and

$$
\begin{aligned}
& \frac{1}{2} \underline{\alpha}_{\mathcal{O}}\left\|\mathbf{u}_{\beta, \mathcal{O}}\right\|_{0,2}^{2}+\frac{c_{\ell}^{3}}{2} \beta\left\|\mathbf{u}_{\beta, \mathcal{O}}\right\|_{0,3}^{3} \leq \frac{1}{2 \underline{\alpha}_{\mathcal{O}}}\left\|\mathbf{g}_{\mathcal{O}}\right\|_{\mathbf{W}(\mathcal{O})^{\prime}}^{2}+\left\|\mathbf{g}_{\mathcal{O}}\right\| \mathbf{W}(\mathcal{O})^{\prime}\|f\|_{0,2}+\left\|f_{\mathcal{O}}\right\|_{0,3}\left\|p_{\beta, \mathcal{O}}\right\|_{0, \frac{3}{2}} \\
& \leq D_{1}+\left\|f_{\mathcal{O}}\right\|_{0,3}\left\|p_{\beta, \mathcal{O}}\right\|_{0, \frac{3}{2}},
\end{aligned}
$$

where $D_{1}$ is a constant depending only on the coefficient $\alpha_{\mathcal{O}}$ and the data functions determining $\mathbf{g}_{\mathcal{O}}$ and $f_{\mathcal{O}}$. Then with the first equation of $\left(\mathcal{P}_{\text {Forch }}\right)$, we obtain, $\forall \mathbf{v} \in \mathbf{W}_{\beta}(\mathcal{O})$,

$$
\begin{aligned}
\left|b_{\mathcal{O}}\left(\mathbf{v}, p_{\beta, \mathcal{O}}\right)\right| & \leq\left|a_{\beta, \mathcal{O}}\left(\mathbf{u}_{\beta, \mathcal{O}}, \mathbf{v}\right)\right|+\left|\mathbf{g}_{\mathcal{O}}(\mathbf{v})\right| \\
& \leq \bar{\alpha}_{\mathcal{O}}\left\|\mathbf{u}_{\beta, \mathcal{O}}\right\|_{0,2}\|\mathbf{v}\|_{0,2}+C_{\ell}^{3} \beta\left\|\mathbf{u}_{\beta, \mathcal{O}}\right\|_{0,3}^{2}\|\mathbf{v}\|_{0,3}+\left\|\mathbf{g}_{\mathcal{O}}\right\| \mathbf{W}(\mathcal{O})^{\prime} \\
& \leq\left(\bar{\alpha}_{\mathcal{O}} C_{L}\|\|_{\mathbf{w}_{\beta, \mathcal{O}}(\mathcal{O})}\left\|_{0,2}+C_{\ell}^{3} \beta\right\| \mathbf{u}_{\beta, \mathcal{O}}\left\|_{0,3}^{2}+\right\| p_{\partial, \mathcal{O}} \|_{\frac{1}{3}, \frac{3}{2}}\right)\|\mathbf{v}\|_{\mathbf{w}_{\beta}(\mathcal{O})},
\end{aligned}
$$

where we again use $C_{L}$, respectively $C_{\ell}$, here specifically for the continuity constant for the embedding $L^{3}(\mathcal{O}) \hookrightarrow$ $L^{2}(\mathcal{O})$, respectively $\ell^{3}\left(R^{d}\right) \hookrightarrow \ell^{2}\left(R^{d}\right)$. Using the inf-sup condition for $b$ on $\mathbf{W}_{\beta}(\mathcal{O}) \times \mathcal{M}_{\beta}(\mathcal{O})$ we have

$$
\theta_{\beta, \mathcal{O}}\|r\|_{0, \frac{3}{2}} \leq \sup _{\mathbf{v} \in \mathbf{W}_{\beta}(\mathcal{O})} \frac{b_{\mathcal{O}}(\mathbf{v}, r)}{\|\mathbf{v}\|_{\mathbf{W}_{\beta}(\mathcal{O})}}
$$

and thus

$$
\theta_{\beta, \mathcal{O}}\left\|p_{\beta, \mathcal{O}}\right\|_{0, \frac{3}{2}} \leq\left(\bar{\alpha}_{\mathcal{O}} C_{L}\left\|\mathbf{u}_{\beta, \mathcal{O}}\right\|_{0,2}+C_{\ell}^{3} \beta\left\|\mathbf{u}_{\beta, \mathcal{O}}\right\|_{0,3}^{2}+\left\|p_{\partial, \mathcal{O}}\right\|_{\frac{1}{3}, \frac{3}{2}}\right) .
$$

Plugging this estimate for $p_{\beta}$ into (4.4) we obtain

$$
\frac{1}{2} \underline{\alpha}_{\mathcal{O}}\left\|\mathbf{u}_{\beta, \mathcal{O}}\right\|_{0,2}^{2}+\frac{c_{\ell}^{3}}{2} \beta\left\|\mathbf{u}_{\beta, \mathcal{O}}\right\|_{0,3}^{3} \leq D_{1}+\frac{1}{\theta_{\beta, \mathcal{O}}}\left\|f_{\mathcal{O}}\right\|_{0,3}\left(\bar{\alpha}_{\mathcal{O}} C_{L}\left\|\mathbf{u}_{\beta, \mathcal{O}}\right\|_{0,2}+C_{\ell}^{3} \beta\left\|\mathbf{u}_{\beta, \mathcal{O}}\right\|_{0,3}^{2}+\left\|p_{\partial, \mathcal{O}}\right\|_{\frac{1}{3}, \frac{3}{2}}\right) .
$$

Now using the inequality

$$
\begin{aligned}
\frac{\bar{\alpha}_{\mathcal{O}} C_{L}}{\theta_{\beta, \mathcal{O}}}\left\|f_{\mathcal{O}}\right\|_{0,3}\left\|\mathbf{u}_{\beta, \mathcal{O}}\right\|_{0,2} & \leq \frac{4}{\underline{\alpha}_{\mathcal{O}}}\left(\frac{\bar{\alpha}_{\mathcal{O}} C_{L}}{\theta_{\beta, \mathcal{O}}}\right)^{2}\left\|f_{\mathcal{O}}\right\|_{0,3}^{2}+\frac{1}{4} \underline{\alpha}_{\mathcal{O}}\left\|\mathbf{u}_{\beta, \mathcal{O}}\right\|_{0,2}^{2} \\
& \leq D_{2}+\frac{1}{4} \underline{\alpha}_{\mathcal{O}}\left\|\mathbf{u}_{\beta, \mathcal{O}}\right\|_{0,2}^{2}
\end{aligned}
$$

it is easy to see that

$$
\begin{aligned}
\frac{1}{4} \underline{\alpha}_{\mathcal{O}}\left\|\mathbf{u}_{\beta, \mathcal{O}}\right\|_{0,2}^{2}+\frac{c_{\ell}^{3}}{2} \beta\left\|\mathbf{u}_{\beta, \mathcal{O}}\right\|_{0,3}^{3} \leq & D_{1}+\frac{4}{\underline{\alpha}_{\mathcal{O}}}\left(\frac{\bar{\alpha}_{\mathcal{O}} C_{L}}{\theta_{\beta, \mathcal{O}}}\right)^{2}\left\|f_{\mathcal{O}}\right\|_{0,3}^{2}+\frac{1}{\theta_{\beta, \mathcal{O}}}\left\|f_{\mathcal{O}}\right\|_{0,3} C_{\ell}^{3} \beta\left\|\mathbf{u}_{\beta, \mathcal{O}}\right\|_{0,3}^{2} \\
& +\frac{1}{\theta_{\beta, \mathcal{O}}}\left\|f_{\mathcal{O}}\right\|_{0,3}\left\|p_{\partial, \mathcal{O}}\right\|_{\frac{1}{3}, \frac{2}{3}} \\
\leq & D_{1}+D_{2}+C_{4} \beta\left\|\mathbf{u}_{\beta, \mathcal{O}}\right\|_{0,3}^{2}+D_{3},
\end{aligned}
$$

with constant terms $D_{2}$, which depends on $f_{\mathcal{O}}, C_{L}, \bar{\alpha}_{\mathcal{O}}, \underline{\alpha}_{\mathcal{O}}$ and $\theta_{\beta, \mathcal{O}}$, and $D_{3}$, which depends on $f_{\mathcal{O}}, p_{\partial, \mathcal{O}}$ and $\theta_{\beta, \mathcal{O}}$, and a constant coefficient $C_{4}$, which depends on $f_{\mathcal{O}}, \theta_{\beta, \mathcal{O}}$ and $C_{\ell}$. Now using Young's inequality, (if $p>0$ and $\frac{1}{p}+\frac{1}{q}=1$ then $\left.a b \leq \frac{a^{p}}{p}+\frac{b^{q}}{q}\right)$ with $a=\left(\beta^{s}\left\|\mathbf{u}_{\beta, \mathcal{O}}\right\|_{0,3}\right)^{2}, b=1, p=\frac{3}{2}$ and $q=3$ one obtains

$$
\frac{1}{4} \underline{\alpha}_{\mathcal{O}}\left\|\mathbf{u}_{\beta, \mathcal{O}}\right\|_{0,2}^{2}+\frac{c_{\ell}^{3}}{2} \beta\left\|\mathbf{u}_{\beta, \mathcal{O}}\right\|_{0,3}^{3} \leq D_{1}+D_{2}+D_{3}+\frac{2}{3} C_{4} \beta^{1-2 s}\left(\beta^{s}\left\|\mathbf{u}_{\beta, \mathcal{O}}\right\|_{0,3}\right)^{3}+\frac{1}{3} C_{4} \beta^{1-2 s}
$$

and that

$$
\frac{1}{4} \underline{\alpha}_{\mathcal{O}}\left\|\mathbf{u}_{\beta, \mathcal{O}}\right\|_{0,2}^{2}+\left(\frac{c_{\ell}^{3}}{2} \beta^{1-3 s}-\frac{2}{3} C_{4} \beta^{1-2 s}\right)\left(\beta^{s}\left\|\mathbf{u}_{\beta, \mathcal{O}}\right\|_{0,3}\right)^{3} \leq D_{1}+D_{2}+D_{3}+\frac{1}{3} C_{4} \beta^{1-2 s}
$$


or, in particular, that (with $s=\frac{1}{2}$ )

$$
\frac{1}{4} \underline{\mathcal{O}}_{\mathcal{O}}\left\|\mathbf{u}_{\beta, \mathcal{O}}\right\|_{0,2}^{2}+\left(\frac{c_{\ell}^{3}}{2} \beta^{-\frac{1}{2}}-\frac{2}{3} C_{4}\right)\left(\beta^{\frac{1}{2}}\left\|\mathbf{u}_{\beta, \mathcal{O}}\right\|_{0,3}\right)^{3} \leq D_{1}+D_{2}+D_{3}+\frac{1}{3} C_{4}:=D_{4} .
$$

Thus for $\beta$ sufficiently small, we obtain an a priori bound on $\underline{\alpha}_{\mathcal{O}}^{\frac{1}{2}}\left\|\mathbf{u}_{\beta, \mathcal{O}}\right\|_{0,2}$ :

$$
\underline{\alpha}_{\mathcal{O}}^{\frac{1}{2}}\left\|\mathbf{u}_{\beta, \mathcal{O}}\right\|_{0,2} \leq 2\left(D_{4}\right)^{\frac{1}{2}}
$$

and also that

$$
\left(\beta^{\frac{1}{2}}\left\|\mathbf{u}_{\beta, \mathcal{O}}\right\|_{0,3}\right)^{3} \leq\left(\frac{c_{\ell}^{3}}{2} \beta^{-\frac{1}{2}}-\frac{2}{3} C_{4}\right)^{-1} D_{4}
$$

so that

$$
\beta^{\frac{1}{2}}\left\|\mathbf{u}_{\beta, \mathcal{O}}\right\|_{0,3} \longrightarrow 0 \quad \text { as } \quad \beta \longrightarrow 0
$$

Rewriting (4.6) as

$$
\frac{1}{4} \underline{\alpha}_{\mathcal{O}}\left\|\mathbf{u}_{\beta, \mathcal{O}}\right\|_{0,2}^{2}+\frac{c_{\ell}^{3}}{2} \beta\left\|\mathbf{u}_{\beta, \mathcal{O}}\right\|_{0,3}^{3} \leq D_{4}+\frac{2}{3} C_{4}\left(\beta^{\frac{1}{2}}\left\|\mathbf{u}_{\beta, \mathcal{O}}\right\|_{0,3}\right)^{3},
$$

we obtain in turn an a priori bound for $\beta^{\frac{1}{3}}\left\|\mathbf{u}_{\beta, \mathcal{O}}\right\|_{0,3}$ :

$$
\beta^{\frac{1}{3}}\left\|\mathbf{u}_{\beta, \mathcal{O}}\right\|_{0,3} \leq\left(D_{4}+\epsilon\right)^{\frac{1}{3}},
$$

with $\epsilon>0$ arbitrarily small for $\beta \leq \bar{\beta}_{\epsilon}$ for some $\bar{\beta}_{\epsilon}>0$. Now combining (4.5), (4.7) and (4.9) one obtains the following a priori bound on $p_{\beta, \mathcal{O}}$ in $L^{\frac{3}{2}}(\mathcal{O})$ :

$$
\left\|p_{\beta, \mathcal{O}}\right\|_{0, \frac{3}{2}} \leq \frac{2 \bar{\alpha}_{\mathcal{O}} C_{L}}{\theta_{\beta, \mathcal{O}} \underline{\alpha}_{\mathcal{O}}^{\frac{1}{2}}}\left(D_{4}\right)^{\frac{1}{2}}+\frac{C_{L}^{3}}{\theta_{\beta, \mathcal{O}}} \beta^{\frac{1}{3}}\left(D_{4}+\epsilon\right)^{\frac{2}{3}}+\frac{1}{\theta_{\beta, \mathcal{O}}}\left\|p_{\partial, \mathcal{O}}\right\|_{\frac{1}{3}, \frac{3}{2}} .
$$

With (4.10), (4.7) and (4.3) the lemma is completed.

From (4.7) and (4.10), we conclude that if $\left\{\beta_{j}\right\}$ is a sequence converging to 0 then there is a subsequence still denoted $\left\{\beta_{j}\right\}$ such that the sequences $\left\{\mathbf{u}_{\beta_{j}, \mathcal{O}}\right\}$ and $\left\{p_{\beta_{j}, \mathcal{O}}\right\}$ are weakly convergent in $\left(L^{2}(\mathcal{O})\right)^{d}$ and in $L^{\frac{3}{2}}(\mathcal{O})$, respectively:

$$
\mathbf{u}_{\beta_{j}, \mathcal{O}} \rightarrow \tilde{\mathbf{u}} \text { in }\left(L^{2}(\mathcal{O})\right)^{d} \quad \text { and } \quad p_{\beta_{j}, \mathcal{O}} \rightarrow \tilde{p} \text { in } L^{\frac{3}{2}}(\mathcal{O})
$$

i.e. explicitly

$$
\int_{\mathcal{O}} \alpha \mathbf{u}_{\beta_{j}, \mathcal{O}} \cdot \mathbf{v} \mathrm{d} x \rightarrow \int_{\mathcal{O}} \alpha \tilde{\mathbf{u}} \cdot \mathbf{v} \mathrm{d} x \forall \mathbf{v} \in\left(L^{2}(\mathcal{O})\right)^{n} \quad \text { and } \quad \int_{\mathcal{O}} p_{\beta_{j}, \mathcal{O}} q \mathrm{~d} x \rightarrow \int_{\mathcal{O}} \tilde{p} q \mathrm{~d} x \forall q \in L^{3}(\mathcal{O}) .
$$

Further, (4.8) implies that $\left\{\beta_{j}^{\frac{1}{2}} \mathbf{u}_{\beta_{j}, \mathcal{O}}\right\}$ converges strongly to 0 in $L^{3}(\mathcal{O})$. Thus

$$
\begin{aligned}
\left|\int_{\mathcal{O}} \beta_{j}\right| \mathbf{u}_{\beta_{j}, \mathcal{O}}\left|\mathbf{u}_{\beta_{j}, \mathcal{O}} \cdot \mathbf{v} \mathrm{d} x\right| & \leq\|\mathbf{v}\|_{0,3}\left\|\beta_{j}\left|\mathbf{u}_{\beta_{j}, \mathcal{O}}\right| \mathbf{u}_{\beta_{j}, \mathcal{O}}\right\|_{0, \frac{3}{2}}=\|\mathbf{v}\|_{0,3}\left(\int_{\mathcal{O}} \beta_{j}^{\frac{3}{2}}\left|\mathbf{u}_{\beta_{j}, \mathcal{O}}\right|^{3} \mathrm{~d} x\right)^{\frac{2}{3}} \\
& =\|\mathbf{v}\|_{0,3}\left\|\beta_{j}^{\frac{1}{2}} \mathbf{u}_{\beta_{j}, \mathcal{O}}\right\|_{0,3}^{2} \rightarrow 0 \text { as } \beta_{j} \rightarrow 0 .
\end{aligned}
$$

Lemma 4.2. Assume that the spatial dimension d satisfies $d \leq 6$. Then the pair $(\tilde{\mathbf{u}}, \tilde{p})$ defined by (4.11) is a solution to $\left(\mathcal{P}_{\text {Darcy }}\right)$ and hence is the unique solution of $\left(\mathcal{P}_{\text {Darcy }}\right): \tilde{\mathbf{u}}=\mathbf{u}_{\mathcal{O}}$ and $\tilde{p}=p_{\mathcal{O}}$. 
Proof. A priori, $\tilde{\mathbf{u}} \in \mathbf{W}_{\beta}(\mathcal{O}) \subset \mathbf{W}(\mathcal{O})$ and $\tilde{p} \in \mathcal{M}_{\beta}(\mathcal{O}) \not \subset \mathcal{M}(\mathcal{O})$. It follows from (4.13) and (4.12) that

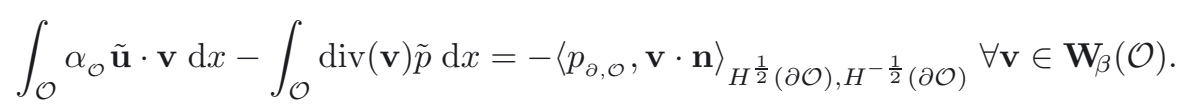

However, if $\mathbf{v} \in \mathcal{D}(\mathcal{O})$, then $\int_{\mathcal{O}} \alpha_{\mathcal{O}} \tilde{\mathbf{u}} \cdot \mathbf{v} \mathrm{d} x-\int_{\mathcal{O}} \operatorname{div}(\mathbf{v}) \tilde{p} \mathrm{~d} x=0$ so that $\nabla \tilde{p}=-\alpha_{\mathcal{O}} \tilde{\mathbf{u}} \in\left(L^{3}(\mathcal{O})\right)^{d}$, and thus $\tilde{p} \in W^{1, \frac{3}{2}}(\mathcal{O})$. By the Sobolev embedding theorem, [1], we have, if $d \leq 6$, then $W^{1, \frac{3}{2}}(\mathcal{O}) \subset L^{2}(\mathcal{O})=\mathcal{M}(\mathcal{O})$. Also we have supposed that $p_{\partial, \mathcal{O}}$ belongs to $W^{\frac{1}{2}, 2}(\partial \mathcal{O})$ as well as to $W^{\frac{1}{3}, \frac{3}{2}}(\partial \mathcal{O})$. Thus each of the terms of $(4.14)$ is well defined for $\mathbf{v} \in \mathbf{W}(\mathcal{O})$, and we have since $\mathbf{W}_{\beta}(\mathcal{O})$ is dense in $\mathbf{W}(\mathcal{O})$ that

$$
\int_{\mathcal{O}} \alpha_{\mathcal{O}} \tilde{\mathbf{u}} \cdot \mathbf{v} \mathrm{d} x-\int_{\mathcal{O}} \operatorname{div}(\mathbf{v}) \tilde{p} \mathrm{~d} x=-\left\langle p_{\partial, \mathcal{O}}, \mathbf{v} \cdot \mathbf{n}\right\rangle_{H^{\frac{1}{2}}(\partial \mathcal{O}), H^{-\frac{1}{2}}(\partial \mathcal{O})} \forall \mathbf{v} \in \mathbf{W}(\mathcal{O}) .
$$

Turning now to the second equation of $\left(\mathcal{P}_{\text {Darcy }}\right)$, we recall that $f_{\mathcal{O}}=q_{\mathcal{O}}$ belongs to $L^{3}(\mathcal{O})=\mathcal{M}_{\beta}(\mathcal{O})^{\prime}$, and thus also to $L^{2}(\mathcal{O})=\mathcal{M}(\mathcal{O})^{\prime}$. As we have seen, the second equation of $\left(\mathcal{P}_{\text {Forch }}\right)$ implies that for each $\beta>0$, $\operatorname{div}\left(\mathbf{u}_{\beta, \mathcal{O}}\right)=f \in L^{3}(\mathcal{O})$. This with (4.7) implies that $\mathbf{u}_{\beta, \mathcal{O}}$ is bounded in the $\mathbf{W}(\mathcal{O})$ norm and that for a subsequence $\left\{\beta_{\ell}\right\}$ of $\left\{\beta_{j}\right\}, \mathbf{u}_{\beta_{\ell}}$ converges weakly to $\tilde{\mathbf{u}}$ in $\mathbf{W}(\mathcal{O})$. It follows that

$$
\int_{\mathcal{O}} \operatorname{div}(\tilde{\mathbf{u}}) r \mathrm{~d} x=\int_{\mathcal{O}} q_{\mathcal{O}} r \forall r \in \mathcal{M}(\mathcal{O})
$$

Thus the pair $(\tilde{\mathbf{u}}, \tilde{p})$ in $\mathbf{W}(\mathcal{O}) \times \mathcal{M}(\mathcal{O})$ is a solution of $\left(\mathcal{P}_{\text {Darcy }}\right)$ and $(\tilde{\mathbf{u}}, \tilde{p})=\left(\mathbf{u}_{\mathcal{O}}, p_{\mathcal{O}}\right)$ by uniqueness.

\section{Darcy as a limit of DarCy-Forchheimer - Domain With a FraCture}

The object of this section is to obtain the original problem $\left(\mathcal{P}\right.$ ) (with Darcy flow in the subdomains $\Omega_{1}$ and $\Omega_{2}$ but Forchheimer flow in the fracture $\gamma$ ) as the limit of the problem $\left(\mathcal{P}_{\beta}\right)$ (with Forcheimer flow in the subdomains and in the fracture) studied in Section 3 when the Forchheimer coefficient in the subdomains $\beta$ decreases to 0. In this section, as in Section 4, for simplicity we shall assume that $\beta_{i}$ is the same constant, positive, real parameter for $i=1$ and $i=2$ :

$$
\beta_{1}=\beta_{2}=\beta>0
$$

(The tensors $\beta_{\gamma}, \alpha_{i}$ and $\alpha_{\gamma}$ (2.4), as well as $\kappa(2.5)$, remain as in Sect. 3.) For each $\beta$ sufficiently small, let $\left(\mathbf{u}_{\beta}, p_{\beta}\right) \in \mathbf{W}_{\beta} \times \mathcal{M}_{\beta}$ be the solution of $\left(\mathcal{P}_{\beta}\right)$. We will derive a priori bounds on $\left(\mathbf{u}_{\beta}, p_{\beta}\right)$ which are independent of $\beta$, thus obtaining a limit function which we shall show is a solution to $(\mathcal{P})$.

Lemma 5.1. There is a constant $C$ independent of $\beta$, such that, for each $\beta$ sufficiently small,

$$
\left\|\mathbf{u}_{\beta}\right\|_{\mathbf{W}}+\left\|p_{\beta}\right\|_{\mathcal{M}_{\beta}}+\beta^{\frac{1}{3}} \sum_{i=1}^{2}\left\|\mathbf{u}_{\beta, i}\right\|_{0,3, \Omega_{i}} \leq C .
$$

In addition,

$$
\beta^{\frac{1}{2}} \sum_{i=1}^{2}\left\|\mathbf{u}_{\beta, i}\right\|_{0,3, \Omega_{i}} \longrightarrow 0, \text { as } \beta \longrightarrow 0
$$


Proof. The proof follows closely the lines of the proof of Lemma 4.1. Taking for test function $\mathbf{v}=\mathbf{u}_{\beta}$ in the first equation of $\left(\mathcal{P}_{\beta}\right)$, noting that $\mathbf{u}_{\beta} \in \mathbf{W}$ and that $\mathbf{g} \in \mathbf{W}^{\prime}$, and letting $C_{\xi}$ denote $\underline{\kappa} \min (1,2 \xi-1)$ we obtain

$$
\begin{aligned}
\sum_{i=1}^{2} & \left(\underline{\alpha}_{i}\left\|\mathbf{u}_{\beta, i}\right\|_{0,2}^{2}+\frac{c_{\ell}^{3}}{2} \beta\left\|\mathbf{u}_{\beta, i}\right\|_{0,3}^{3}\right)+\underline{\alpha}_{\gamma}\left\|\mathbf{u}_{\beta, \gamma}\right\|_{0,2}^{2}+\frac{c_{\ell}^{3}}{2} \underline{\beta}_{\gamma}\left\|\mathbf{u}_{\beta, \gamma}\right\|_{0,3}^{3}+\sum_{i=1}^{2} C_{\xi}\left\|\mathbf{u}_{\beta, i} \cdot \mathbf{n}\right\|_{0,2}^{2} \\
& \leq a_{\beta}\left(\mathbf{u}_{\beta}, \mathbf{u}_{\beta}\right)=\mathbf{g}\left(\mathbf{u}_{\beta}\right)+b_{\beta}\left(\mathbf{u}_{\beta}, p_{\beta}\right) \\
& \leq\|\mathbf{g}\|_{\mathbf{W}^{\prime}}\left\|\mathbf{u}_{\beta}\right\|_{\mathbf{W}}+\left\|\operatorname{Div} \mathbf{u}_{\beta}\right\|_{\mathcal{M}_{\beta}^{\prime}}\left\|p_{\beta}\right\|_{\mathcal{M}_{\beta}} \\
& =\|\mathbf{g}\|_{\mathbf{W}^{\prime}}\left(\left\|\mathbf{u}_{\beta}\right\|_{\mathbf{v}}+\left\|\operatorname{Div} \mathbf{u}_{\beta}\right\|_{\mathcal{M}^{\prime}}+\sum_{i=1}^{2}\left\|\mathbf{u}_{\beta, i} \cdot \mathbf{n}\right\|_{0,2, \gamma}\right)+\left\|\operatorname{Div} \mathbf{u}_{\beta}\right\|_{\mathcal{M}_{\beta}^{\prime}}\left\|p_{\beta}\right\|_{\mathcal{M}_{\beta}},
\end{aligned}
$$

and from the second equation we have $\operatorname{Div} \mathbf{u}_{\beta}=f$ so that

$$
\begin{aligned}
\left\|\operatorname{Div} \mathbf{u}_{\beta}\right\|_{\mathcal{M}_{\beta}^{\prime}} & =\|f\|_{\mathcal{M}_{\beta}^{\prime}} \\
\| \operatorname{Div} \mathbf{u}_{\beta} & \left\|_{\mathcal{M}^{\prime}}=\right\| f \|_{\mathcal{M}^{\prime}} .
\end{aligned}
$$

Combining these estimates, analogously to (4.4) we obtain

$$
\begin{aligned}
& \sum_{i=1}^{2}\left(\underline{\alpha}_{i}\left\|\mathbf{u}_{\beta, i}\right\|_{0,2}^{2}+\frac{c_{\ell}^{3}}{2} \beta\left\|\mathbf{u}_{\beta, i}\right\|_{0,3}^{3}\right)+\underline{\alpha}_{\gamma}\left\|\mathbf{u}_{\beta, \gamma}\right\|_{0,2}^{2}+\frac{c_{\ell}^{3}}{2} \underline{\beta}_{\gamma}\left\|\mathbf{u}_{\beta, \gamma}\right\|_{0,3}^{3}+\sum_{i=1}^{2} C_{\xi}\left\|\mathbf{u}_{\beta, i} \cdot \mathbf{n}\right\|_{0,2}^{2} \\
& \quad \leq\|\mathbf{g}\|_{\mathbf{w}^{\prime}}\left(\left\|\mathbf{u}_{\beta}\right\|_{\mathbf{v}}+\|f\|_{\mathcal{M}^{\prime}}+\sum_{i=1}^{2}\left\|\mathbf{u}_{\beta, i} \cdot \mathbf{n}\right\|_{0,2, \gamma}\right)+\|f\|_{\mathcal{M}_{\beta}^{\prime}}\left\|p_{\beta}\right\|_{\mathcal{M}_{\beta}} \\
& \quad \leq\|\mathbf{g}\| \mathbf{w}^{\prime}\left(\sum_{i=1}^{2}\left\|\mathbf{u}_{\beta, i}\right\|_{0,2}+\left\|\mathbf{u}_{\beta, \gamma}\right\|_{0,3, \gamma}+\sum_{i=1}^{2}\left\|\mathbf{u}_{\beta, i} \cdot \mathbf{n}\right\|_{0,2, \gamma}\right)+\|f\|_{\mathcal{M}_{\beta}^{\prime}}\left\|p_{\beta}\right\|_{\mathcal{M}_{\beta}}+D_{1},
\end{aligned}
$$

where the constant term $D_{1}$ depends on $\mathbf{g}$ and on $f$. Then, using Young's inequality we have

$$
\begin{aligned}
& \sum_{i=1}^{2}\left(\frac{1}{2} \underline{\alpha}_{i}\left\|\mathbf{u}_{\beta, i}\right\|_{0,2}^{2}+\frac{c_{\ell}^{3}}{2} \beta\left\|\mathbf{u}_{\beta, i}\right\|_{0,3}^{3}\right)+\underline{\alpha}_{\gamma}\left\|\mathbf{u}_{\beta, \gamma}\right\|_{0,2}^{2}+\frac{2}{3} \frac{c_{\ell}^{3}}{2} \underline{\beta}_{\gamma}\left\|\mathbf{u}_{\beta, \gamma}\right\|_{0,3}^{3}+\frac{1}{2} \sum_{i=1}^{2} C_{\xi}\left\|\mathbf{u}_{\beta, i} \cdot \mathbf{n}\right\|_{0,2}^{2} \\
& \leq\|f\|_{\mathcal{M}_{\beta}^{\prime}}\left\|p_{\beta}\right\|_{\mathcal{M}_{\beta}}+D_{1}+D_{2}
\end{aligned}
$$

where $D_{2}$ depends on $\mathbf{g}, \underline{\alpha}_{i}, \underline{\beta}_{\gamma}$, and $C_{\xi}$. The inf-sup condition for $b_{\beta}: \mathbf{W}_{\beta} \times \mathcal{M}_{\beta} \longrightarrow R$ together with the first equation of $\left(\mathcal{P}_{\beta}\right)$ yields

$$
\begin{aligned}
\theta_{\beta}\left\|p_{\beta}\right\|_{\mathcal{M}_{\beta}} & \leq \sup _{\mathbf{v} \in \mathbf{W}_{\beta}} \frac{b\left(\mathbf{v}, p_{\beta}\right)}{\|\mathbf{v}\|_{\mathbf{w}_{\beta}}} \\
& \leq \sup _{\mathbf{v} \in \mathbf{W}_{\beta}} \frac{a_{\beta}\left(\mathbf{u}_{\beta}, \mathbf{v}\right)-\mathbf{g}(\mathbf{v})}{\|\mathbf{v}\|_{\mathbf{W}_{\beta}}}
\end{aligned}
$$

and using (3.1)

$$
\begin{aligned}
\left|a_{\beta}\left(\mathbf{u}_{\beta}, \mathbf{v}\right)-\mathbf{g}(\mathbf{v})\right| \leq & \sum_{i=1}^{2}\left(\bar{\alpha}_{i}\left\|\mathbf{u}_{\beta, i}\right\|_{0,2}\left\|\mathbf{v}_{i}\right\|_{0,2}+C_{\ell}^{3} \beta\left\|\mathbf{u}_{\beta, i}\right\|_{0,3}^{2}\left\|\mathbf{v}_{i}\right\|_{0,3}\right) \\
& +\bar{\kappa} \xi\left(\sum_{i=1}^{2}\left\|\mathbf{u}_{\beta, i} \cdot \mathbf{n}\right\|_{0,2, \gamma}\right)\left(\sum_{i=1}^{2}\left\|\mathbf{v}_{i} \cdot \mathbf{n}\right\|_{0,2, \gamma}\right) \\
& +\bar{\alpha}_{\gamma}\left\|\mathbf{u}_{\beta, \gamma}\right\|_{0,2, \gamma}\left\|\mathbf{v}_{\gamma}\right\|_{0,2, \gamma}+C_{\ell}^{2} \bar{\beta}_{\gamma}\left\|\mathbf{u}_{\beta, \gamma}\right\|_{0,3, \gamma}^{2}\left\|\mathbf{v}_{\gamma}\right\|_{0,3, \gamma}+\|\mathbf{g}\|_{\mathbf{w}_{\beta}^{\prime}}\|\mathbf{v}\|_{\mathbf{w}_{\beta}} .
\end{aligned}
$$


Then combining the last two estimates, analogously to (4.5) we have

$$
\begin{aligned}
\theta_{\beta}\left\|p_{\beta}\right\|_{\mathcal{M}_{\beta}} \leq & \sum_{i=1}^{2}\left(C_{L} \bar{\alpha}_{i}\left\|\mathbf{u}_{\beta, i}\right\|_{0,2}+C_{\ell}^{3} \beta\left\|\mathbf{u}_{\beta, i}\right\|_{0,3}^{2}+\bar{\kappa} \xi\left\|\mathbf{u}_{\beta, i} \cdot \mathbf{n}\right\|_{0,2, \gamma}\right) \\
& +C_{L} \bar{\alpha}_{\gamma}\left\|\mathbf{u}_{\beta, \gamma}\right\|_{0,2, \gamma}+C_{\ell}^{2} \bar{\beta}_{\gamma}\left\|\mathbf{u}_{\beta, \gamma}\right\|_{0,3, \gamma}^{2}+\|\mathbf{g}\|_{\mathbf{w}_{\beta}^{\prime}} .
\end{aligned}
$$

So

$$
\begin{aligned}
\sum_{i=1}^{2}( & \left.\frac{1}{2} \underline{\alpha}_{i}\left\|\mathbf{u}_{\beta, i}\right\|_{0,2}^{2}+\frac{c_{\ell}^{3}}{2} \beta\left\|\mathbf{u}_{\beta, i}\right\|_{0,3}^{3}\right)+\underline{\alpha}_{\gamma}\left\|\mathbf{u}_{\beta, \gamma}\right\|_{0,2}^{2}+\frac{c_{\ell}^{3}}{3} \underline{\beta}_{\gamma}\left\|\mathbf{u}_{\beta, \gamma}\right\|_{0,3}^{3}+\frac{1}{2} \sum_{i=1}^{2} C_{\xi}\left\|\mathbf{u}_{\beta, i} \cdot \mathbf{n}\right\|_{0,2}^{2} \\
\leq & D_{1}+D_{2}+D_{3}+\frac{\|f\|_{\mathcal{M}_{\beta}^{\prime}}}{\theta_{\beta}}\left(\sum_{i=1}^{2}\left(C_{L} \bar{\alpha}_{i}\left\|\mathbf{u}_{\beta, i}\right\|_{0,2}+C_{\ell}^{3} \beta\left\|\mathbf{u}_{\beta, i}\right\|_{0,3}^{2}+\bar{\kappa} \xi\left\|\mathbf{u}_{\beta, i} \cdot \mathbf{n}\right\|_{0,2, \gamma}\right)\right. \\
& \left.+C_{L} \bar{\alpha}_{\gamma}\left\|\mathbf{u}_{\beta, \gamma}\right\|_{0,2, \gamma}+C_{\ell}^{2} \bar{\beta}_{\gamma}\left\|\mathbf{u}_{\beta, \gamma}\right\|_{0,3, \gamma}^{2}\right),
\end{aligned}
$$

where $D_{3}$ depends on $\mathbf{g}, \theta_{\beta}$, and $f$. Then using Young's inequality (three times with exponents 2 and 2 and twice with exponents 3 and $\frac{3}{2}$ ) we obtain

$$
\begin{aligned}
\sum_{i=1}^{2}\left(\frac{1}{4} \underline{\alpha}_{i}\left\|\mathbf{u}_{\beta, i}\right\|_{0,2}^{2}+\frac{c_{\ell}^{3}}{2} \beta\left\|\mathbf{u}_{\beta, i}\right\|_{0,3}^{3}\right)+ & \frac{1}{2} \underline{\alpha}_{\gamma}\left\|\mathbf{u}_{\beta, \gamma}\right\|_{0,2}^{2}+\frac{c_{\ell}^{3}}{3} \underline{\beta}_{\gamma}\left\|\mathbf{u}_{\beta, \gamma}\right\|_{0,3}^{3}+\frac{1}{4} \sum_{i=1}^{2} C_{\xi}\left\|\mathbf{u}_{\beta, i} \cdot \mathbf{n}\right\|_{0,2}^{2} \\
& \leq D_{1}+D_{2}+D_{3}+D_{4}+\frac{2}{3} C_{5} \sum_{i=1}^{2}\left(\beta^{\frac{1}{2}}\left\|\mathbf{u}_{\beta, i}\right\|_{0,3}\right)^{3}
\end{aligned}
$$

with $D_{4}$ depending on $\bar{\alpha}_{i}, \underline{\alpha}_{i}, \bar{\alpha}_{\gamma}, \underline{\alpha}_{\gamma}, \bar{\beta}_{\gamma}, \underline{\beta}_{\gamma}, \bar{\kappa}, \underline{\kappa}, \xi, \theta_{\beta}$, and $f$, and, analogously to (4.6),

$$
\begin{aligned}
\sum_{i=1}^{2}\left(\frac{1}{4} \underline{\alpha}_{i}\left\|\mathbf{u}_{\beta, i}\right\|_{0,2}^{2}\right. & \left.+\left(\frac{c_{\ell}^{3}}{2} \beta^{-\frac{1}{2}}-\frac{2}{3} C_{5}\right)\left(\beta^{\frac{1}{2}}\left\|\mathbf{u}_{\beta, i}\right\|_{0,3}\right)^{3}+\frac{1}{4} C_{\xi}\left\|\mathbf{u}_{\beta, i} \cdot \mathbf{n}\right\|_{0,2}^{2}\right)+\frac{1}{4} \underline{\alpha}_{\gamma}\left\|\mathbf{u}_{\beta, \gamma}\right\|_{0,2}^{2} \\
& +\frac{c_{\ell}^{3}}{3} \underline{\beta}_{\gamma}\left\|\mathbf{u}_{\beta, \gamma}\right\|_{0,3}^{3} \leq D_{1}+D_{2}+D_{3}+D_{4} .
\end{aligned}
$$

(Recall that $\theta_{\beta}$ does not depend on $\beta$.) Hence

$$
\beta^{\frac{1}{2}}\left\|\mathbf{u}_{\beta, i}\right\|_{0,3} \longrightarrow 0 \text { as } \beta \longrightarrow 0, \quad i=1,2,
$$

as in (4.8), and, as in (4.7), (4.9) each of the terms $\left\|\mathbf{u}_{\beta, i}\right\|_{0,2},\left\|\mathbf{u}_{\beta, i} \cdot \mathbf{n}\right\|_{0,2, \gamma},\left\|\mathbf{u}_{\beta, \gamma}\right\|_{0,2, \gamma},\left\|\mathbf{u}_{\beta, \gamma}\right\|_{0,3, \gamma}$ and $\beta^{\frac{1}{3}}\left\|\mathbf{u}_{\beta, i}\right\|_{0,3}$ is bounded by a positive constant $D_{5}$, depending on $\bar{\alpha}_{i}, \bar{\alpha}_{\gamma}, \bar{\beta}_{\gamma}, \bar{\kappa}, \xi, f$ and $\mathbf{g}$ but independent of $\beta$ :

$$
\left\|\mathbf{u}_{\beta, i}\right\|_{0,2}+\left\|\mathbf{u}_{\beta, i} \cdot \mathbf{n}\right\|_{0,2, \gamma}+\left\|\mathbf{u}_{\beta, \gamma}\right\|_{0,2, \gamma}+\left\|\mathbf{u}_{\beta, \gamma}\right\|_{0,3, \gamma}+\beta^{\frac{1}{3}}\left\|\mathbf{u}_{\beta, i}\right\|_{0,3} \leq D_{5}
$$

Combining (5.1) and (5.4) yields an a priori bound on $\mathbf{u}_{\beta, i}$ in the $H\left(\operatorname{div}, \Omega_{i}\right)$-norm. Equation (5.1) also gives an a priori bound on $\operatorname{div} \mathbf{u}_{\beta, \gamma}-\left[\mathbf{u}_{\beta_{j}, 1} \cdot \mathbf{n}-\mathbf{u}_{\beta_{j}, 2} \cdot \mathbf{n}\right]$ in the $L^{3}(\gamma)$-norm, which completes the a priori bound of $\left\|\mathbf{u}_{\beta}\right\|_{\mathbf{w}}$.

To bound $p_{\beta}$ we recall (5.2)

$$
\begin{aligned}
\theta_{\beta}\left(\sum_{i=1}^{2}\left\|p_{\beta, i}\right\|_{0, \frac{3}{2}}+\left\|p_{\beta, \gamma}\right\|_{0, \frac{3}{2}, \gamma}\right) \leq & \sum_{i=1}^{2}\left(C_{L} \bar{\alpha}_{i}\left\|\mathbf{u}_{\beta, i}\right\|_{0,2}+C_{\ell}^{3} \beta\left\|\mathbf{u}_{\beta, i}\right\|_{0,3}^{2}+\xi \bar{\kappa}\left\|\mathbf{u}_{\beta, i} \cdot \mathbf{n}\right\|_{0,2, \gamma}\right) \\
& +C_{L} \bar{\alpha}_{\gamma}\left\|\mathbf{u}_{\beta, \gamma}\right\|_{0,2, \gamma}+C_{\ell}^{2} \bar{\beta}_{\gamma}\left\|\mathbf{u}_{\beta, \gamma}\right\|_{0,3, \gamma}^{2}+\|\mathbf{g}\|_{\mathbf{w}_{\beta}^{\prime}}
\end{aligned}
$$


and obtain for a positive constant $D_{6}$, depending on $\bar{\alpha}_{i}, \bar{\alpha}_{\gamma}, \bar{\beta}_{\gamma}, \bar{\kappa}, \xi, f$, $\mathbf{g}$ and $\theta_{\beta}$ but independent of $\beta$ :

$$
\sum_{i=1}^{2}\left\|p_{\beta, i}\right\|_{0, \frac{3}{2}}+\left\|p_{\beta, \gamma}\right\|_{0, \frac{3}{2}, \gamma} \leq D_{6},
$$

which gives the a priori bound on $\left\|p_{\beta}\right\|_{\mathcal{M}_{\beta}}$.

Theorem 5.2. Suppose $d \leq 6$. There exists a unique solution $(\mathbf{u}, p) \in \mathbf{W} \times \mathcal{M}$ of problem $(\mathcal{P})$, and $(\mathbf{u}, p)$ is a weak limit of solutions $\left(\mathbf{u}_{\beta}, p_{\beta}\right) \in \mathbf{W} \times \mathcal{M}$ in the sense made precise below.

Proof. The proof follows from the error bounds (5.4), (5.5) obtained in Lemma 5.1. As the spaces $H\left(\operatorname{div}, \Omega_{i}\right)$, $L^{\frac{3}{2}}\left(\Omega_{i}\right), L^{3}(\gamma), L^{\frac{3}{2}}(\gamma)$ and $L^{2}(\gamma)$ are reflexive Banach spaces they are sequentially weakly compact. Thus from (4.7) and (4.10), we conclude that if $\left\{\beta_{\ell}\right\}$ is a sequence converging to 0 then there is a subsequence $\left\{\beta_{j}\right\}$ such that the sequences $\left\{\mathbf{u}_{\beta_{j}, i}\right\},\left\{p_{\beta_{j}, i}\right\},\left\{\mathbf{u}_{\beta_{j}, \gamma}\right\},\left\{p_{\beta_{j}, \gamma}\right\},\left\{\mathbf{u}_{\beta_{j}, i} \cdot \mathbf{n}\right\}$ and $\operatorname{div} \mathbf{u}_{\beta_{j}, \gamma}-\left[\mathbf{u}_{\beta_{j}, 1} \cdot \mathbf{n}-\mathbf{u}_{\beta_{j}, 2} \cdot \mathbf{n}\right]$ are weakly convergent in $H\left(\operatorname{div}, \Omega_{i}\right), L^{\frac{3}{2}}\left(\Omega_{i}\right), L^{3}(\gamma), L^{\frac{3}{2}}(\gamma), L^{2}(\gamma)$ and in $L^{3}(\gamma)$ respectively:

$$
\begin{aligned}
& \mathbf{u}_{\beta_{j}, i} \rightarrow \tilde{\mathbf{u}}_{i} \text { in } H\left(\operatorname{div}, \Omega_{i}\right) \quad p_{\beta_{j}, i} \rightarrow \tilde{p}_{i} \text { in } L^{\frac{3}{2}}\left(\Omega_{i}\right) \\
& \mathbf{u}_{\beta_{j}, \gamma} \rightarrow \tilde{\mathbf{u}}_{\gamma} \text { in } L^{3}(\gamma) \quad p_{\beta_{j}, \gamma} \rightarrow \tilde{p}_{\gamma} \text { in } L^{\frac{3}{2}}(\gamma) \\
& \operatorname{div} \mathbf{u}_{\beta_{j}, \gamma}-\left[\mathbf{u}_{\beta_{j}, 1} \cdot \mathbf{n}-\mathbf{u}_{\beta_{j}, 2} \cdot \mathbf{n}\right] \rightarrow \hat{\mathbf{u}}_{\gamma} \text { in } L^{3}(\gamma) \quad \mathbf{u}_{\beta_{j}, i} \cdot \mathbf{n} \rightarrow \hat{\mathbf{u}}_{i} \text { in } L^{2}(\gamma)
\end{aligned}
$$

and

$$
\beta^{\frac{1}{2}} \mathbf{u}_{\beta, i} \longrightarrow 0 \text { in } L^{3}\left(\Omega_{i}\right)
$$

We remark that since $\left\|\mathbf{u}_{\beta, i} \cdot \mathbf{n}\right\|_{H^{-\frac{1}{2}\left(\partial \Omega_{i}\right)}} \leq C\left\|\mathbf{u}_{\beta, i}\right\|_{H\left(\operatorname{div}, \Omega_{i}\right)}$ is bounded independently of $\beta$ that $\mathbf{u}_{\beta_{j}, i} \cdot \mathbf{n}$ converges weakly to $\tilde{\mathbf{u}}_{i} \cdot \mathbf{n}$ in $H^{-\frac{1}{2}}\left(\partial \Omega_{i}\right)$. Then since $\mathbf{u}_{\beta_{j}, i} \cdot \mathbf{n}$ converges weakly to $\hat{\mathbf{u}}_{i}$ in $L^{2}(\gamma)$, we have $\hat{\mathbf{u}}_{i}=\tilde{\mathbf{u}}_{i} \cdot \mathbf{n}$

We also note that $\operatorname{div} \mathbf{u}_{\beta_{j}, \gamma} \in L^{2}(\gamma)$ so that $\mathbf{u}_{\beta_{j}, \gamma} \in H(\operatorname{div}, \gamma)$. Further, $\left\|\operatorname{div} \mathbf{u}_{\beta_{j}, \gamma}\right\|_{L^{2}(\gamma)}$ and thus $\left\|\mathbf{u}_{\beta_{j}, \gamma}\right\|_{H(\text { div }, \gamma)}$ is bounded independently of $\beta$ so that $\mathbf{u}_{\beta_{j}, \gamma}$ converges weakly to $\tilde{\mathbf{u}}_{\gamma}$ in $H(\operatorname{div}, \gamma)$. Following the same lines of reasoning we conclude that

$$
\hat{\mathbf{u}}_{\gamma}=\operatorname{div} \tilde{\mathbf{u}}_{\gamma}-\left(\tilde{\mathbf{u}}_{1} \cdot \mathbf{n}-\tilde{\mathbf{u}}_{2} \cdot \mathbf{n}\right) .
$$

Thus with $\tilde{\mathbf{u}}=\left(\tilde{\mathbf{u}}_{1}, \tilde{\mathbf{u}}_{2}, \tilde{\mathbf{u}}_{\gamma}\right)$ we have $\tilde{\mathbf{u}} \in \mathbf{W}$, and it is clear that

$$
b(\tilde{\mathbf{u}}, r)=\langle\operatorname{Div} \tilde{\mathbf{u}}, r\rangle_{\mathcal{M}^{\prime}, \mathcal{M}}=f(r), \quad \forall r \in \mathcal{M},
$$

i.e. the second equation of $(\mathcal{P})$ is satisfied by $\tilde{\mathbf{u}}$.

For each $\beta>0$, the first equation of $\left(\mathcal{P}_{\beta}\right)$ is

$$
\begin{aligned}
& a_{\beta}\left(\mathbf{u}_{\beta}, \mathbf{v}\right)-b\left(\mathbf{v}, p_{\beta}\right)=\mathbf{g}(\mathbf{v}), \quad \forall \mathbf{v} \in \mathbf{W}_{\beta}, \\
& \sum_{i=1}^{2} \int_{\Omega_{i}}\left(\alpha_{i}+\beta\left|\mathbf{u}_{\beta, i}\right|\right) \mathbf{u}_{\beta, i} \cdot \mathbf{v}_{i} \mathrm{~d} x+\int_{\gamma}\left(\alpha_{\gamma}+\beta_{\gamma}\left|\mathbf{u}_{\beta, \gamma}\right|\right) \mathbf{u}_{\beta, \gamma} \cdot \mathbf{v}_{\gamma} \mathrm{d} s \\
& +\sum_{i=1}^{2} \int_{\gamma} \frac{1}{\kappa}\left(\xi \mathbf{u}_{\beta, i} \cdot \mathbf{n}+\bar{\xi} \mathbf{u}_{\beta, i+1} \cdot \mathbf{n}\right) \mathbf{v}_{i} \cdot \mathbf{n} \mathrm{d} s-b\left(\mathbf{v}, p_{\beta}\right)=\mathbf{g}(\mathbf{v}), \quad \forall \mathbf{v} \in \mathbf{W}_{\beta},
\end{aligned}
$$

or

$$
\begin{aligned}
\sum_{i=1}^{2} \int_{\Omega_{i}} \beta\left|\mathbf{u}_{\beta, i}\right| \mathbf{u}_{\beta, i} \cdot \mathbf{v}_{i} \mathrm{~d} x+\int_{\gamma} \beta_{\gamma}\left|\mathbf{u}_{\beta, \gamma}\right| \mathbf{u}_{\beta, \gamma} \cdot \mathbf{v}_{\gamma} \mathrm{d} s=-\sum_{i=1}^{2} \int_{\Omega_{i}} \alpha_{i} \mathbf{u}_{\beta, i} \cdot \mathbf{v}_{i} \mathrm{~d} x-\int_{\gamma} \alpha_{\gamma} \mathbf{u}_{\beta, \gamma} \cdot \mathbf{v}_{\gamma} \mathrm{d} s \\
-\sum_{i=1}^{2} \int_{\gamma} \frac{1}{\kappa}\left(\xi \mathbf{u}_{\beta, i} \cdot \mathbf{n}+\bar{\xi} \mathbf{u}_{\beta, i+1} \cdot \mathbf{n}\right) \mathbf{v}_{i} \cdot \mathbf{n} \mathrm{d} s+b\left(\mathbf{v}, p_{\beta}\right)+\mathbf{g}(\mathbf{v}), \quad \forall \mathbf{v} \in \mathbf{W}_{\beta}
\end{aligned}
$$


Then taking the limit as $\beta$ goes to 0 we have, due to (3.1) and Lemma 5.1

$$
\begin{aligned}
\lim _{\beta \rightarrow 0} \int_{\gamma} \beta_{\gamma}\left|\mathbf{u}_{\beta, \gamma}\right| \mathbf{u}_{\beta, \gamma} \cdot \mathbf{v}_{\gamma} \mathrm{d} s=-\sum_{i=1}^{2} \int_{\Omega_{i}} \alpha_{i} \tilde{\mathbf{u}}_{i} \cdot \mathbf{v}_{i} \mathrm{~d} x-\int_{\gamma} \alpha_{\gamma} \tilde{\mathbf{u}}_{\gamma} \cdot \mathbf{v}_{\gamma} \mathrm{d} s & \\
& -\sum_{i=1}^{2} \int_{\gamma} \frac{1}{\kappa}\left(\xi \tilde{\mathbf{u}}_{i} \cdot \mathbf{n}+\bar{\xi} \tilde{\mathbf{u}}_{i+1} \cdot \mathbf{n}\right) \mathbf{v}_{i} \cdot \mathbf{n} \mathrm{d} s+b(\mathbf{v}, \tilde{p})+\mathbf{g}(\mathbf{v}), \quad \forall \mathbf{v} \in \mathbf{W}_{\beta},
\end{aligned}
$$

and in particular, for test functions $\mathbf{v} \in\left(\mathcal{D}\left(\Omega_{1}\right)\right)^{d} \times\left(\mathcal{D}\left(\Omega_{2}\right)\right)^{d} \times\{0\}$,

$$
\sum_{i=1}^{2} \int_{\Omega_{i}} \alpha_{i} \tilde{\mathbf{u}}_{i} \cdot \mathbf{v}_{i} \mathrm{~d} x-\sum_{i=1}^{2} \int_{\Omega_{i}} \operatorname{div}_{i} \tilde{p}_{i} \mathrm{~d} x=0 .
$$

Thus $\nabla \tilde{p}_{i}=-\alpha_{i} \tilde{\mathbf{u}}_{i} \in L^{2}\left(\Omega_{i}\right)$ and therefore $\tilde{p}_{i} \in W_{1, \frac{3}{2}}\left(\Omega_{i}\right)$. From the Sobolev embedding theorem we then have $\tilde{p}_{i} \in L^{2}\left(\Omega_{i}\right)$ (for $d \leq 6$ ) which means that $\tilde{p} \in \mathcal{M}$. Now from the density of $\mathbf{W}_{\beta}$ in $\mathbf{W}$ we conclude that

$$
\begin{aligned}
& \lim _{\beta \rightarrow 0} \int_{\gamma} \beta_{\gamma}\left|\mathbf{u}_{\beta, \gamma}\right| \mathbf{u}_{\beta, \gamma} \cdot \mathbf{v}_{\gamma} \mathrm{d} s=-\sum_{i=1}^{2} \int_{\Omega_{i}} \alpha_{i} \tilde{\mathbf{u}}_{i} \cdot \mathbf{v}_{i} \mathrm{~d} x-\int_{\gamma} \alpha_{\gamma} \tilde{\mathbf{u}}_{\gamma} \cdot \mathbf{v}_{\gamma} \mathrm{d} s \\
&-\sum_{i=1}^{2} \int_{\gamma} \frac{1}{\kappa}\left(\xi \tilde{\mathbf{u}}_{i} \cdot \mathbf{n}+\bar{\xi} \tilde{\mathbf{u}}_{i+1} \cdot \mathbf{n}\right) \mathbf{v}_{i} \cdot \mathbf{n} \mathrm{d} s+b(\mathbf{v}, \tilde{p})+\mathbf{g}(\mathbf{v}), \quad \forall \mathbf{v} \in \mathbf{W} .
\end{aligned}
$$

Now there remains to see that

$$
\lim _{\beta \rightarrow 0} \int_{\gamma} \beta_{\gamma}\left|\mathbf{u}_{\beta, \gamma}\right| \mathbf{u}_{\beta, \gamma} \cdot \mathbf{v}_{\gamma} \mathrm{d} s=\int_{\gamma} \beta_{\gamma}\left|\tilde{\mathbf{u}}_{\gamma}\right| \tilde{\mathbf{u}}_{\gamma} \cdot \mathbf{v}_{\gamma} \mathrm{d} s, \quad \forall \mathbf{v}_{\gamma} \in L^{3}(\gamma) .
$$

Toward this end we define a mapping on $L^{3}(\gamma) \times L^{3}(\gamma)$ by

$$
\left(\mathbf{w}_{\gamma}, \mathbf{v}_{\gamma}\right) \mapsto \int_{\gamma} \beta_{\gamma}\left|\mathbf{w}_{\gamma}\right| \mathbf{w}_{\gamma} \cdot \mathbf{v}_{\gamma} \mathrm{d} s
$$

and the associated mapping $C: L^{3}(\gamma) \longrightarrow L^{\frac{3}{2}}(\gamma)$. That the mapping $C$ is monotone and continuous can be shown as in the proof of Lemma 3.1 where the monotonicity and continuity of $A_{\beta}$ are shown. Therefore $C$ maps weakly convergent sequences to convergent sequences; see [20]. Thus, since $\mathbf{u}_{\beta, \gamma} \rightarrow \tilde{\mathbf{u}}_{\gamma}$, we have that $C\left(\mathbf{u}_{\beta, \gamma}\right) \rightarrow C\left(\tilde{\mathbf{u}}_{\gamma}\right)$ in $L^{3}(\gamma)$ which now yields that

$$
a(\tilde{\mathbf{u}}, \mathbf{v})-b(\mathbf{v}, \tilde{p})=\mathbf{g}(\mathbf{v}), \quad \forall \mathbf{v} \in \mathbf{W} .
$$

Thus $(\tilde{\mathbf{u}}, \tilde{p}) \in \mathbf{W} \times \mathcal{M}$ is a solution of $(\mathcal{P})$.

As in (3.5) we see that $A$ is strictly monotone on $\mathbf{W}$. Thus we can refer to Lemma A.1 for uniqueness.

\section{ApPENDix A}

In this appendix for the sake of completeness we give the definition and some basic properties of the spaces $W^{p}(\operatorname{div}, \Omega)$, and we include the demonstrations of some lemmas needed in the previous sections.

\section{A.1. The spaces $W^{p}(\operatorname{div}, \mathcal{O})$}

We recall the definition given in $[15,18]$ of the spaces $W^{p}(\operatorname{div}, \mathcal{O})$ for $\mathcal{O} \subset R^{d}$ a bounded domain in $R^{d}$ and $p \in R$ a number with $1 \leq p$ :

$$
W^{p}(\operatorname{div}, \mathcal{O}):=\left\{\mathbf{v} \in\left(L^{p}(\mathcal{O})\right)^{d}: \operatorname{div} \mathbf{v} \in L^{p}(\mathcal{O})\right\}
$$


with norm

$$
\|\mathbf{v}\|_{W^{p}(\operatorname{div}, \mathcal{O})}:=\|\mathbf{v}\|_{L^{p}(\mathcal{O})}+\|\operatorname{div} \mathbf{v}\|_{L^{p}(\mathcal{O})} .
$$

As pointed out in [15] and in [18] it suffices to note that $W^{p}(\operatorname{div}, \mathcal{O})$ is a closed subset of $\left(L^{p}(\mathcal{O})\right)^{d}$ to see that $W^{p}(\operatorname{div}, \mathcal{O})$ is a reflexive Banach space. Further, normal traces of elements of $W^{p}(\operatorname{div}, \mathcal{O})$ belong to $W^{-\frac{1}{p}, p}(\partial \mathcal{O})$.

\section{A.2. A general uniqueness result}

The object here is to show a uniqueness result that is used in the proofs of Theorems 3.4 and 5.2: that if $A$, $B$ and $B^{t}$ are the operators associated with a mixed formulation and $A$ is strictly monotone and $B$ is surjective, then the mixed problem has no more than one solution; more precisely

Lemma A.1. Let $X$ and $Y$ be Hilbert spaces and let a be a form, linear in its second variable, on $Y \times Y$ and $b$ a bilinear form on $Y \times X$ and let $A: Y \longrightarrow Y^{\prime}$ and $B: Y \longrightarrow X^{\prime}$ be the associated linear operators defined by $\langle A(v), w\rangle_{Y^{\prime}, Y}=a(v, w), \forall w \in Y$ and $\langle B(v), r\rangle_{X^{\prime}, X}=b(v, r), \forall r \in X$, respectively. Suppose further that $f \in X^{\prime}$ and $g \in Y^{\prime}$. Then if $A$ is strictly monotone and $B$ is surjective, the problem

Find $u \in Y$ and $p \in X$ such that

$$
\begin{aligned}
a(u, v)-b(v, p) & =g(v) \forall v \in Y \\
b(u, r) & =f(r) \forall r \in X
\end{aligned}
$$

has at most one solution.

Proof. Suppose that $(u, p)$ and $(w, s) \in Y \times X$ are solutions to $(\mathcal{P})$. Then

$$
\begin{aligned}
& a(u, v)-a(w, v)-b(v, p-s)=0, \forall v \in Y \\
& b(u-w, r) \quad=0, \forall r \in X
\end{aligned}
$$

and taking as test functions $v=u-w$ and $r=p-s$ we obtain $a(u, u-w)-a(w, u-w)=0$ or $\langle A(u)-A(w), u-$ $w\rangle_{Y^{\prime}, Y}=0$. Then, as $A$ is strictly monotone we have $u=w$. To see that $p=s$ we suppose the contrary and use the surjectivity of $B$ to obtain an element $v \in Y$ with $\langle B(v), p-s\rangle_{X^{\prime}, X} \neq 0$. However, as $u=w$ we have $0=a(u, v)-a(w, v)=b(v, p-s)=\langle B(v), p-s\rangle_{X^{\prime}, X}$ contradicting the choice of $v$.

\section{A.3. Some inf-sup conditions}

In this paragraph we give proofs of the fact that some of the bilinear operators considered in the text satisfy the inf-sup condition.

Proof of Lemma 3.2. This proof is just as that for the problem with Darcy flow in the fracture and in the subdomain (see the proof of [16], Thm. 4.1), only modified for the inf sup condition in the $W^{3}(\operatorname{div}) \times L^{\frac{3}{2}}$ setting as in the proof of ([15], Lem. A.3). It clearly suffices to show that the induced mapping $B_{\beta}=$ Div : $\mathbf{W}_{\beta} \longrightarrow \mathcal{M}_{\beta}^{\prime}$ is surjective and has a continuous right inverse. Given an element $\psi=\left(\psi_{1}, \psi_{2}, \psi_{\gamma}\right) \in \mathcal{M}_{\beta}^{\prime}$, to construct an element $\mathbf{v}_{\psi}=\left(\mathbf{v}_{1}, \mathbf{v}_{2}, \mathbf{v}_{\gamma}\right) \in \mathbf{W}_{\beta}$ with $B_{\beta} \mathbf{v}_{\psi}=\psi$ and $\left\|\mathbf{v}_{\psi}\right\|_{\mathbf{w}_{\beta}} \leq C\|\psi\|_{\mathcal{M}_{\beta}^{\prime}}$ one solves the auxiliary problem $\Delta \phi=\hat{\psi}$ in $\Omega, \phi=0$ on $\Gamma$, with right hand side $\hat{\psi} \in L^{3}(\Omega)$, the function that agrees with $\psi_{i}$ on $\Omega_{i}$. The solution $\phi$ is in $W^{2,3}(\Omega)$ if $\Omega$ is sufficiently regular (otherwise we solve the same homogenous Dirichlet problem on a larger more regular domain and take the restriction of the solution to $\Omega$ ), and $\|\phi\|_{2,3, \Omega} \leq C\|\hat{\psi}\|_{0,3, \Omega}$ with a constant $C$ that depends only on $\Omega$. Then $\hat{\mathbf{v}}:=\nabla \phi \in\left(W^{1,3}(\Omega)\right)^{d} \subset W^{3}(\operatorname{div}, \Omega)$ and $\operatorname{div} \hat{\mathbf{v}}=\hat{\psi} \in L^{3}(\Omega)$ so that $\|\hat{\mathbf{v}}\|_{W^{3}(\operatorname{div}, \Omega)} \leq(1+d C)\|\hat{\psi}\|_{0,3, \Omega}$. We also have $\mathbf{v}_{i}:=\hat{\mathbf{v}}_{\mid \Omega_{i}} \in\left(W^{1,3}\left(\Omega_{i}\right)\right)^{d} \subset W^{3}\left(\operatorname{div}, \Omega_{i}\right)$ with $\operatorname{div} \mathbf{v}_{i}=\psi_{i} \in$ $L^{3}\left(\Omega_{i}\right)$ so that

$$
\left\|\mathbf{v}_{i}\right\|_{W^{3}\left(\operatorname{div}, \Omega_{i}\right)} \leq(1+d C)\|\hat{\psi}\|_{0,3, \Omega} \leq(1+d C) \frac{1}{c_{\ell}}\left(\left\|\psi_{1}\right\|_{0,3, \Omega_{1}}+\left\|\psi_{2}\right\|_{0,3, \Omega_{2}}\right) \leq \widetilde{C}\|\psi\|_{\mathcal{M}_{\beta}^{\prime}} .
$$


As $\mathbf{v}_{i} \in\left(W^{1,3}\left(\Omega_{i}\right)\right)^{d}$, we have $\mathbf{v}_{i} \cdot \mathbf{n}_{i} \in W^{\frac{2}{3}, 3}\left(\partial \Omega_{i}\right)$, where $\mathbf{n}_{i}$ is the exterieur unit normal vector on $\Omega_{i}$, and it follows that $\mathbf{v}_{i} \cdot \mathbf{n}_{i} \in L^{3}(\gamma) \subset L^{2}(\gamma)$ and

$$
\left\|\mathbf{v}_{i} \cdot \mathbf{n}_{i}\right\|_{0,2, \gamma} \leq\left\|\mathbf{v}_{i} \cdot \mathbf{n}_{i}\right\|_{\frac{2}{3}, 3, \partial \Omega_{i}} \leq\left\|\mathbf{v}_{i}\right\|_{W^{3}\left(\operatorname{div}, \Omega_{i}\right)} \leq \widetilde{C}\|\psi\|_{\mathcal{M}_{\beta}^{\prime}} .
$$

Thus the pair $\left(\mathbf{v}_{1}, \mathbf{v}_{2}\right)$ is suitable for the first two components of $\mathbf{v}$.

To obtain the third component $\mathbf{v}_{\gamma}$, note that as $\hat{\mathbf{v}} \in W^{3}(\operatorname{div}, \Omega)$ we have $\mathbf{v}_{1} \cdot \mathbf{n}_{1}+\mathbf{v}_{2} \cdot \mathbf{n}_{2}=0$ on $\gamma$, and thus the problem in the fracture domain $\gamma$ is decoupled from that in the subdomains $\Omega_{1}$ and $\Omega_{2}$. One has only to define $\mathbf{v}_{\gamma}:=\nabla \phi_{\gamma}$ where $\phi_{\gamma}$ is the solution of $\Delta \phi_{\gamma}=\psi_{\gamma}$ in $\gamma, \phi_{\gamma}=0$ on $\partial \gamma$. It is straightforward to verify now that $\mathbf{v}_{\psi}=\left(\mathbf{v}_{1}, \mathbf{v}_{2}, \mathbf{v}_{\gamma}\right)$ is a suitable antecedent for $\psi$ and that the mapping $\psi \mapsto \mathbf{v}_{\psi}$ is continuous from $\mathcal{M}_{\beta}^{\prime}$ into $\mathbf{W}_{\beta}$.

Lemma A.2. The inf-sup condition holds for the bilinear form $b: \mathbf{W} \times \mathcal{M} \longrightarrow R$; i.e. there exists $\theta \in R$ such that for each $r \in \mathcal{M}$

$$
\sup _{\mathbf{v} \in \mathbf{W}} \frac{b(\mathbf{v}, r)}{\|\mathbf{v}\|_{\mathbf{W}}} \geq \theta\|r\|_{\mathcal{M}}
$$

Proof. The proof of this lemma is just as that of Lemma 3.2 only the auxiliary problem in the subdomains is in the $H(\operatorname{div}) \times L^{2}$ setting. As the auxiliary problems in the subdomains and in the fracture domain decouple no difficulty arises from the fact that one of these involves $H(\operatorname{div}) \times L^{2}$ while the other involves $W^{3}(\operatorname{div}) \times L^{\frac{3}{2}}$.

Acknowledgements. We are grateful to the anonymous referee, who pointed out a considerable simplification of the original proof of Theorem 1.

\section{REFERENCES}

[1] R. Adams, Sobolev Spaces, vol. 65 of Pure and Appl. Math. Academic Press, New York (1975).

[2] C. Alboin, J. Jaffré, J. Roberts and C. Serres, Domain decomposition for flow in porous media with fractures, in Proc. of the 11th Int. Conf. on Domain Decomposition Methods in Greenwich, England (1999).

[3] G. Allaire, Homogenization of the stokes flow in a connected porous medium. Asymptotic Anal. 2 (1989) $203-222$.

[4] G. Allaire, One-phase newtonian flow, in Homogenization and Porous Media, vol. 6 of Interdisciplinary Appl. Math., edited by U. Hornung. Springer-Verlag, New York (1997) 45-69.

[5] Y. Amirat, Ecoulements en milieu poreux n'obeissant pas a la loi de darcy. RAIRO Modél. Math. Anal. Numér. 25 (1991) 273-306.

[6] P. Angot, F. Boyer and F. Hubert, Asymptotic and numerical modelling of flows in fractured porous media. ESAIM: M2AN 43 (2009) 239-275.

[7] M. Balhoff, A. Mikelic and M. Wheeler, Polynomial filtration laws for low reynolds number flows through porous media. Transport in Porous Media (2009).

[8] J. Bear, Dynamics of Fluids in Porous Media. American Elsevier Pub. Co., New York (1972).

[9] F. Brezzi, On the existence, uniqueness and approximation of saddle-point problems arising from lagrangian multipliers. RAIRO: Modél. Math. Anal. Numér. 8 (1974) 129-151.

[10] P. Fabrie, Regularity of the solution of Darcy-Forchheimer's equation. Nonlinear Anal., Theory Methods Appl. 13 (1989) 1025-1049.

[11] I. Faille, E. Flauraud, F. Nataf, S. Pegaz-Fiornet, F. Schneider and F. Willien, A new fault model in geological basin modelling, application to finite volume scheme and domain decomposition methods, in Finie Volumes for Complex Appl. III. Edited by R. Herbin and D. Kroner. Hermés Penton Sci. (2002) 543-550.

[12] P. Forchheimer, Wasserbewegung durch Boden. Z. Ver. Deutsch. Ing. 45 (1901) 1782-1788.

[13] N. Frih, J. Roberts and A. Saada, Un modèle darcy-frochheimer pour un écoulement dans un milieu poreux fracturé. ARIMA 5 (2006) 129-143.

[14] N. Frih, J. Roberts and A. Saada, Modeling fractures as interfaces: a model for forchheimer fractures. Comput. Geosci. 12 (2008) 91-104.

[15] P. Knabner and G. Summ, Solvability of the mixed formulation for Darcy-Forchheimer flow in porous media. Submitted.

[16] V. Martin, J. Jaffré and J.E. Roberts, Modeling fractures and barriers as interfaces for flow in porous media. SIAM J. Sci. Comput. 26 (2005) 1667-1691. 
[17] R. Showalter and F. Morales, The narrow fracture approximation by channeled flow. J. Math. Anal. Appl. 365 (2010) $320-331$.

[18] G. Summ, Lösbarkeit un Diskretisierung der gemischten Formulierung für Darcy-Frochheimer-Fluss in porösen Medien. Ph.D. thesis. Friedrich-Alexander-Universität Erlangen-Nürnberg (2001).

[19] L. Tartar, Convergence of the homogenization process, in Non-homogeneous Media and Vibration Theory, vol. 127 of Lect. Notes Phys. Edited by E. Sancez-Palencia. Springer-Verlag (1980).

[20] E. Zeidler, Nonlinear function anaysis and its applications - Nonlinear monotone operators. Springer-Verlag, Berlin, Heidelberg, New York (1990). 